\title{
TILTING THEORY FOR GORENSTEIN RINGS IN DIMENSION ONE
}

\author{
RAGNAR-OLAF BUCHWEITZ ${ }^{1, \dagger}$, OSAMU IYAMA $^{2}$ and KOTA YAMAURA ${ }^{3}$ \\ ${ }^{1}$ Department of Computer and Mathematical Sciences, University of Toronto Scarborough, \\ Toronto, Ontario, Canada M1C 1A4 \\ ${ }^{2}$ Graduate School of Mathematics, Nagoya University, Furocho, Chikusaku, \\ Nagoya 464-8602, Japan; \\ email: iyama@math.nagoya-u.ac.jp \\ ${ }^{3}$ Graduate Faculty of Interdisciplinary Research, Faculty of Engineering, University of Yamanashi, \\ Takeda, Kofu 400-8510, Japan; \\ email: kyamaura@yamanashi.ac.jp
}

Received 24 September 2019; accepted 20 April 2020

\begin{abstract}
In representation theory, commutative algebra and algebraic geometry, it is an important problem to understand when the triangulated category $\mathrm{D}_{\mathrm{sg}}^{\mathbb{Z}}(R)=\underline{\mathrm{CM}}_{0}^{\mathbb{Z}} R$ admits a tilting (respectively, silting) object for a $\mathbb{Z}$-graded commutative Gorenstein ring $R=\bigoplus_{i \geqslant 0} R_{i}$. Here $\mathrm{D}_{\mathrm{sg}}^{\mathbb{Z}}(R)$ is the singularity category, and $\underline{C M}_{0}^{\mathbb{Z}} R$ is the stable category of $\mathbb{Z}$-graded Cohen-Macaulay (CM) $R$-modules, which are locally free at all nonmaximal prime ideals of $R$.

In this paper, we give a complete answer to this problem in the case where $\operatorname{dim} R=1$ and $R_{0}$ is a field. We prove that $\underline{\mathrm{CM}}_{0}^{\mathbb{Z}} R$ always admits a silting object, and that $\underline{\mathrm{CM}}_{0}^{\mathbb{Z}} R$ admits a tilting object if and only if either $R$ is regular or the $a$-invariant of $R$ is nonnegative. Our silting/tilting object will be given explicitly. We also show that if $R$ is reduced and nonregular, then its $a$-invariant is nonnegative and the above tilting object gives a full strong exceptional collection in $\underline{\mathrm{CM}}_{0}^{\mathbb{Z}} R=\underline{\mathrm{CM}}^{\mathbb{Z}} R$.
\end{abstract}

2010 Mathematics Subject Classification: 13C14 (primary); 16E35 (secondary)

\section{Introduction}

1.1. Background. The study of maximal Cohen-Macaulay (CM) modules is one of the central subjects in commutative algebra and representation

\footnotetext{
$\dagger$ The first author passed away on November 11, 2017.

(c) The Author(s), 2020. Published by Cambridge University Press. This is an Open Access article, distributed under the terms of the Creative Commons Attribution licence (http://creativecommons.org/licenses/by/4.0/), which permits unrestricted re-use, distribution, and reproduction in any medium, provided the original work is properly cited.
} 
theory $[5,14,54,66,72]$. When the ring $R$ is Gorenstein, the category

$$
\mathrm{CM} R=\left\{X \in \bmod R \mid \operatorname{Ext}_{R}^{i}(X, R)=0 \text { for all } i \geqslant 1\right\}
$$

of CM $R$-modules forms a Frobenius category, and therefore its stable category $\underline{\mathrm{CM}} R$ has a natural structure of a triangulated category [28]. The Verdier quotient $\mathrm{D}_{\mathrm{sg}}(R)=\mathrm{D}^{\mathrm{b}}(\bmod R) / \mathrm{K}^{\mathrm{b}}($ proj $R)$ introduced by Buchweitz [11] and Orlov [61] is canonically triangle equivalent to $\underline{\mathrm{CM} R}$, and hence is enhanced by the Frobenius category CM $R$. When $R$ is a hypersurface, it is also triangle equivalent to the stable category of matrix factorizations [20]. It has increasing importance in algebraic geometry and physics.

Tilting theory controls triangle equivalences between derived categories of rings and plays a significant role in various areas of mathematics (see for example, [3]). Tilting theory also gives a powerful tool to study the stable categories of Gorenstein rings. For example, for a finite-dimensional algebra $\Lambda$ of finite global dimension, there is a triangle equivalence

$$
\underline{\bmod }^{\mathbb{Z}} T(\Lambda) \simeq \mathrm{K}^{\mathrm{b}}(\operatorname{proj} \Lambda)
$$

for the stable category $\bmod ^{\mathbb{Z}} T(\Lambda)$ of the $\mathbb{Z}$-graded modules over the trivial extension algebra $T(\Lambda)$ [28]. This is an important result, which gives a large family of representation-finite self-injective algebras (see for example, [67]). The second classical example is a triangle equivalence

$$
\underline{\bmod }^{\mathbb{Z}} \bigwedge\left(k^{n}\right) \simeq \mathrm{K}^{\mathrm{b}}(\operatorname{proj} \Lambda)
$$

for the exterior algebra $\bigwedge\left(k^{n}\right)$ and the Beilinson algebra $\Lambda[8,9]$. The third classical example is a triangle equivalence

$$
\underline{\mathrm{CM}}^{\mathbb{Z}} R \simeq \mathrm{K}^{\mathrm{b}}(\bmod k Q)
$$

for the stable category of $\mathbb{Z}$-graded $C M$ modules over a $\mathbb{Z}$-graded simple surface singularity $R$ and the path algebra $k Q$ of the Dynkin quiver $Q$ of the same type $[22,23,45]$. Each of the above triangle equivalences follows from the fact that the stable category has a tilting object (see Definition 4.1). In fact, under mild assumptions, a triangulated category admits a tilting object if and only if it is triangle equivalent to $\mathrm{K}^{\mathrm{b}}$ (proj $\Lambda$ ) for some ring $\Lambda$ (Proposition 4.2). Recently, the class of silting objects was introduced to complete the class of tilting objects in the study of t-structures [49] and mutation [1]. We will see that they also play an important role in the study of the stable categories of Gorenstein rings.

It is well known in CM representation theory that the subcategory $\mathrm{CM}_{0} R=\left\{X \in \mathrm{CM} R \mid X_{\mathfrak{p}} \in \operatorname{proj} R_{\mathfrak{p}}\right.$ for all $\mathfrak{p} \in \operatorname{Spec} R$ with $\left.\operatorname{dim} R_{\mathfrak{p}}<\operatorname{dim} R\right\}$ 
behaves much nicer than CM $R$ since it enjoys Auslander-Reiten-Serre duality, and hence it has almost split sequences if $R$ is complete local [5, 72] (cf. Proposition 4.6). Therefore, for a $\mathbb{Z}$-graded Gorenstein ring $R$, we consider the Frobenius category

$$
\mathrm{CM}_{0}^{\mathbb{Z}} R:=\left\{X \in \bmod ^{\mathbb{Z}} R \mid X \in \mathrm{CM}_{0} R \text { as an ungraded } R \text {-module }\right\} .
$$

There are a number of $\mathbb{Z}$-graded Gorenstein rings $R$ such that the stable categories $\underline{\mathrm{CM}}_{0}^{\mathbb{Z}} R$ admit tilting objects; see for example, $[2,15,16,21,24,25,31,33,38,39$, 44-46, 50-53, 55, 59, 68-71] and a survey article [36]. The following problem is important in representation theory, commutative algebra and algebraic geometry.

Problem 1.1. Let $R=\bigoplus_{i \geqslant 0} R_{i}$ be a $\mathbb{Z}$-graded Gorenstein ring such that $R_{0}$ is a field. When does the stable category $\underline{\mathrm{CM}}_{0}^{\mathbb{Z}} R$ of $\mathbb{Z}$-graded CM $R$-modules have a tilting object?

When $\operatorname{dim} R=0, \underline{\mathrm{CM}}_{0}^{\mathbb{Z}} R=\underline{\bmod }^{\mathbb{Z}} R$ always has a tilting object. In fact, the third author gave a much more general result [71], which also implies the triangle equivalence (1.1) as a special case.

The aim of this paper is to give a complete answer to Problem 1.1 when $\operatorname{dim} R=1$. Surprisingly to us, it is determined by the $a$-invariant of $R$. Our results are summarized as follows.

THEOREM 1.2 (Theorems 1.4 and 1.6). Let $R=\bigoplus_{i \geqslant 0} R_{i}$ be a Gorenstein ring in dimension one such that $R_{0}$ is a field. Then $\underline{\mathrm{CM}}_{0}^{\mathbb{Z}} R$ always has a silting object. Moreover, $\mathrm{CM}_{0}^{\mathbb{Z}} R$ has a tilting object if and only if either $R$ is regular or the $a$-invariant of $R$ is nonnegative.

In particular, the Grothendieck group $K_{0}\left(\underline{\mathrm{CM}}_{0}^{\mathbb{Z}} R\right)$ is a free abelian group of finite rank (Corollary 1.7). To prove Theorem 1.2, we interpret $\underline{\mathrm{CM}}_{0}^{\mathbb{Z}} R$ as a thick subcategory of the singularity category $\mathrm{D}_{\mathrm{sg}}^{\mathbb{Z}}(R)=\mathrm{D}^{\mathrm{b}}\left(\bmod ^{\mathbb{Z}} R\right) / \mathrm{K}^{\mathrm{b}}\left(\operatorname{proj}^{\mathbb{Z}} R\right)$ (Proposition 4.8) and give analogues of Orlov's semiorthogonal decompositions [62] of $\mathrm{D}^{\mathrm{b}}\left(\bmod ^{\mathbb{Z}} R\right)$ (Theorem 3.1).

1.2. Our results. Throughout this subsection, we assume the following.

(R1) $R$ is a $\mathbb{Z}$-graded commutative Gorenstein ring of Krull dimension one.

(R2) $R=\bigoplus_{i \geqslant 0} R_{i}$ and $k:=R_{0}$ is a field.

Let $S$ be the set of all homogeneous non-zero-divisors in $R$, and $K:=R S^{-1}$ the $\mathbb{Z}$-graded total quotient ring of $R$. There exists then an integer $p>0$ such 
that $K(p) \simeq K$ as a graded $R$-module (Lemma 4.11(b)). Moreover, $\operatorname{dim} R=1$ implies that $K=R\left[r^{-1}\right]$ holds for each homogeneous non-zero-divisor $r$ of positive degree (Lemma 4.10).

Let $\bmod ^{\mathbb{Z}} R$ be the category of $\mathbb{Z}$-graded finitely generated $R$-modules, $\bmod _{0}^{\mathbb{Z}} R$ be the category of $\mathbb{Z}$-graded $R$-modules of finite length, and $\operatorname{proj}^{\mathbb{Z}} R$ be the category of $\mathbb{Z}$-graded finitely generated projective $R$-modules. For $X \in \bmod ^{\mathbb{Z}} R$ and $n \in \mathbb{Z}$, let

$$
X_{\geqslant n}=X_{>n-1}:=\bigoplus_{i \geqslant n} X_{i}
$$

Let qgr $R=\bmod ^{\mathbb{Z}} R / \bmod _{0}^{\mathbb{Z}} R$ be the quotient category. This is equivalent to the category of coherent sheaves on the quotient stack [(Spec $\left.\left.R \backslash\left\{R_{>0}\right\}\right) / k^{*}\right]$ [62, Proposition 2.17]. Let $\mathrm{D}^{\mathrm{b}}$ (qgr $R$ ) be the bounded derived category of qgr $R$, and let per(qgr $R$ ) be its thick subcategory generated by $\operatorname{proj}^{\mathbb{Z}} R$. Our starting point is the following result on the geometric side, where we refer to [35] for the notion of exceptional collections.

THEOREM 1.3. Under the setting (R1) and (R2), the following holds true.

(a) qgr $R$ has a progenerator $U:=\bigoplus_{i=1}^{p} K(i)_{\geqslant 0}=\bigoplus_{i=1}^{p} K_{\geqslant i}(i)$, and $\operatorname{per}(\mathrm{qgr} R)$ has a tilting object $U$.

(b) We have an equivalence $\operatorname{qgr} R \simeq \bmod \Lambda$ and a triangle equivalence $\operatorname{per}(\mathrm{qgr} R) \simeq \mathrm{K}^{\mathrm{b}}(\operatorname{proj} \Lambda)$ for $\Lambda:=\operatorname{End}_{\mathrm{qgr} R}(U)$.

(c) We have

$$
\Lambda \simeq \operatorname{End}_{R}^{\mathbb{Z}}(U)=\left[\begin{array}{ccccc}
K_{0} & K_{-1} & \cdots & K_{2-p} & K_{1-p} \\
K_{1} & K_{0} & \cdots & K_{3-p} & K_{2-p} \\
\vdots & \vdots & \ddots & \vdots & \vdots \\
K_{p-2} & K_{p-3} & \cdots & K_{0} & K_{-1} \\
K_{p-1} & K_{p-2} & \cdots & K_{1} & K_{0}
\end{array}\right]
$$

(d) $\Lambda$ is a finite-dimensional self-injective k-algebra.

(e) If $R$ is reduced, then $\Lambda$ is a semisimple k-algebra. Otherwise, $\Lambda$ has infinite global dimension.

(f) If $R$ is reduced, then any ordering in the isomorphism classes of indecomposable direct summands of $U$ gives a full strong exceptional collection in $\operatorname{per}(\mathrm{ggr} R)$. Otherwise, $\operatorname{per}(\mathrm{qgr} R)$ does not have a full strong exceptional collection. 
Now we discuss tilting objects on the algebraic side. Just as we were considering per $(q g r R)$ on the geometric side rather than $\mathrm{D}^{\mathrm{b}}(\mathrm{ggr} R)$, we consider the subcategory $\mathrm{CM}_{0}^{\mathbb{Z}} R$ of $\mathrm{CM}^{\mathbb{Z}} R$ in (1.3). This can be described as

$$
\mathrm{CM}_{0}^{\mathbb{Z}} R=\left\{X \in \mathrm{CM}^{\mathbb{Z}} R \mid K \otimes_{R} X \in \operatorname{proj} K\right\}
$$

(Proposition 4.15). Moreover, $\mathrm{CM}_{0}^{\mathbb{Z}} R=\mathrm{CM}^{\mathbb{Z}} R$ holds if and only if $R$ is reduced.

There exists an integer $a \in \mathbb{Z}$ such that $\operatorname{Ext}_{R}^{1}(k, R(a)) \simeq k$ in $\bmod ^{\mathbb{Z}} R$. We call $a$ the $a$-invariant (-a the Gorenstein parameter) of $R[10,26]$. It can be characterized as the smallest integer $a$ such that $R_{>a}=K_{>a}$ (Lemma 4.11(a)). When $R$ has a nonnegative $a$-invariant, $\mathrm{CM}_{0}^{\mathbb{Z}} R$ always has a tilting object by the following result.

THEOREM 1.4. Under the setting (R1) and (R2), assume moreover that the ainvariant a of $R$ is nonnegative. Then the following holds true.

(a) $\underline{\mathrm{CM}}_{0}^{\mathbb{Z}} R$ has a tilting object

$$
V:=\bigoplus_{i=1}^{a+p} R(i)_{\geqslant 0}=\bigoplus_{i=1}^{a+p} R_{\geqslant i}(i) .
$$

(b) We have a triangle equivalence $\underline{\mathrm{CM}}_{0}^{\mathbb{Z}} R \simeq \mathrm{K}^{\mathrm{b}}(\operatorname{proj} \Gamma)$ for $\Gamma:=\underline{\operatorname{End}}_{R}^{\mathbb{Z}}(V)$.

(c) We have $\Gamma \simeq \operatorname{End}_{R}^{\mathbb{Z}}(V)$. Moreover, these algebras are isomorphic to

$$
\left[\begin{array}{cccccccccc}
R_{0} & 0 & \cdots & 0 & 0 & 0 & 0 & \cdots & 0 & 0 \\
R_{1} & R_{0} & \cdots & 0 & 0 & 0 & 0 & \cdots & 0 & 0 \\
\vdots & \vdots & \ddots & \vdots & \vdots & \vdots & \vdots & \cdots & \vdots & \vdots \\
R_{a-2} & R_{a-3} & \cdots & R_{0} & 0 & 0 & 0 & \cdots & 0 & 0 \\
R_{a-1} & R_{a-2} & \cdots & R_{1} & R_{0} & 0 & 0 & \cdots & 0 & 0 \\
K_{a} & K_{a-1} & \cdots & K_{2} & K_{1} & K_{0} & K_{-1} & \cdots & K_{2-p} & K_{1-p} \\
K_{a+1} & K_{a} & \cdots & K_{3} & K_{2} & K_{1} & K_{0} & \cdots & K_{3-p} & K_{2-p} \\
\vdots & \vdots & \vdots & \vdots & \vdots & \vdots & \vdots & \ddots & \vdots & \vdots \\
K_{a+p-2} & K_{a+p-3} & \cdots & K_{p} & K_{p-1} & K_{p-2} & K_{p-3} & \cdots & K_{0} & K_{-1} \\
K_{a+p-1} & K_{a+p-2} & \cdots & K_{p+1} & K_{p} & K_{p-1} & K_{p-2} & \cdots & K_{1} & K_{0}
\end{array}\right] .
$$

(d) $\Gamma$ is an Iwanaga-Gorenstein $k$-algebra, that is, inj.dim $\Gamma_{\Gamma}=\operatorname{inj} \cdot \operatorname{dim}_{\Gamma} \Gamma$ $<\infty$.

(e) $R$ is reduced if and only if $\Gamma$ has finite global dimension. 
The above $V$ is an analogue of the tilting object in $\underline{\bmod }^{\mathbb{Z}} A$ given in [71] for a $\mathbb{Z}$-graded finite-dimensional self-injective algebra.

As a special case of Theorem 1.4, we obtain the following result for reduced rings.

COROLLARY 1.5. Under the setting (R1) and (R2), assume moreover that $R$ is reduced and not regular. Then the following holds true.

(a) The a-invariant a of $R$ is nonnegative.

(b) $\mathrm{CM}^{\mathbb{Z}} R$ has a tilting object

$$
V:=\bigoplus_{i=1}^{a+p} R(i)_{\geqslant 0}=\bigoplus_{i=1}^{a+p} R_{\geqslant i}(i) .
$$

(c) We have a triangle equivalence $\underline{\mathrm{CM}}^{\mathbb{Z}} R \simeq \mathrm{D}^{\mathrm{b}}(\bmod \Gamma)$, where $\Gamma:=\underline{\operatorname{End}}_{R}^{\mathbb{Z}}(V)$ is a finite-dimensional $k$-algebra with finite global dimension.

(d) There exists an ordering in the isomorphism classes of indecomposable direct summands of $V$, which forms a full strong exceptional collection in $\underline{\mathrm{CM}}^{\mathbb{Z}} R$.

Note that, for the case of hypersurfaces, a different tilting object with a much nicer endomorphism algebra was constructed in [30] before this paper.

Now we discuss the case when $R$ has a negative $a$-invariant. In this case, the following result shows that $\underline{\mathrm{CM}}_{0}^{\mathbb{Z}} R$ never has a tilting object except for the trivial case, where we denote by thick $P$ the smallest thick subcategory containing $P$. We refer to Section 2.4 for a concrete example.

THEOREM 1.6. Under the setting (R1) and (R2), assume moreover that the ainvariant a of $R$ is negative. Then the following holds true.

(a) $\underline{\mathrm{CM}}_{0}^{\mathbb{Z}} R$ has a silting object $\bigoplus_{i=1}^{a+p} R(i)_{\geqslant 0}$.

(b) We have a triangle equivalence $\mathrm{CM}_{0}^{\mathbb{Z}} R \simeq \mathrm{K}^{\mathrm{b}}(\operatorname{proj} \Lambda) /$ thick $P$, where $\Lambda$ is given by (1.4) and $P$ is the projective $\Lambda$-module corresponding to the first -a rows.

(c) $\underline{\mathrm{CM}}_{0}^{\mathbb{Z}} R$ has a tilting object if and only if $R$ is regular.

As an application of our results, we calculate the Grothendieck groups of the triangulated categories per(qgr $R$ ) and $\underline{\mathrm{CM}}_{0}^{\mathbb{Z}} R$. We decompose $K$ into a product $K=K^{1} \times \cdots \times K^{m}$ of rings $K^{i}$, which are ring-indecomposable. For each $1 \leqslant$ $i \leqslant m$, let $p_{i}$ be the smallest positive integer satisfying $K^{i}\left(p_{i}\right) \simeq K^{i}$ in $\bmod ^{\mathbb{Z}} K$. 
COROLLARY 1.7. Under the setting (R1) and (R2), the following holds true.

(a) The Grothendieck group of per(qgr $R)$ is a free abelian group of rank $\sum_{i=1}^{m} p_{i}$.

(b) The Grothendieck group of $\underline{\mathrm{CM}}_{0}^{\mathbb{Z}} R$ is a free abelian group of rank $a+$ $\sum_{i=1}^{m} p_{i}$.

Another application is the following observation, which shows that our category $\mathrm{CM}_{0}^{\mathbb{Z}} R$ is a rich source of triangulated categories.

COROLLARY 1.8. Let $A$ be a $\mathbb{Z}$-graded commutative artinian Gorenstein ring such that $A=A_{\geqslant 0}$ and $A_{0}$ is a field. Then there exists a ring $R$ satisfying (R1) and (R2) such that $\underline{\mathrm{CM}}_{0}^{\mathbb{Z}} R$ is triangle equivalent to $\mathrm{K}^{\mathrm{b}}\left(\mathrm{proj}^{\mathbb{Z} / a \mathbb{Z}} A\right)$, where $a$ is the $a$-invariant of $A$ and we regard $A$ as a $(\mathbb{Z} / a \mathbb{Z})$-graded ring naturally.

Conventions. All modules are right modules. The composition of morphisms (respectively, arrows) $f: X \rightarrow Y$ and $g: Y \rightarrow Z$ is denoted by $g f$. We denote by $k$ an arbitrary field.

\section{Examples}

2.1. Hypersurface singularities. In this subsection, we study hypersurface singularities in dimension one with standard grading. In the rest, let $k$ be an arbitrary field,

$$
R=k[x, y] /(f) \quad \text { with } \operatorname{deg} x=\operatorname{deg} y=1 \text {, and } \Gamma=\underline{\operatorname{End}}_{R}^{\mathbb{Z}}(V)
$$

for the tilting object $V$ given in Theorem 1.4. Then $a=n-2$ holds for $n:=\operatorname{deg} f$, and there is a triangle equivalence

$$
\underline{\mathrm{CM}}_{0}^{\mathbb{Z}} R \simeq \mathrm{K}^{\mathrm{b}}(\operatorname{proj} \Gamma) .
$$

We show that $\Gamma$ has self-injective dimension at most 2 and possibly infinite global dimension. More precisely, we prove the following results in Section 4.6.

THEOREM 2.1. Under the above setting, the following holds true.

(a) $\Gamma$ is an Iwanaga-Gorenstein $k$-algebra with inj.dim $\Gamma_{\Gamma}=$ inj. $\operatorname{dim}_{\Gamma} \Gamma \leqslant 2$.

(b) Assume $n \geqslant 4$. Then there is no Iwanaga-Gorenstein k-algebra $\Gamma^{\prime}$ that is derived equivalent to $\Gamma$ and satisfies inj.dim $\Gamma_{\Gamma^{\prime}}^{\prime}=\operatorname{inj} \cdot \operatorname{dim}_{\Gamma^{\prime}} \Gamma^{\prime} \leqslant 1$. 
In the rest, we assume $f=\prod_{i=1}^{m} f_{i}^{n_{i}}$, where $f_{i}=\alpha_{i} x+\beta_{i} y$ is a linear form such that $\left(f_{i}\right) \neq\left(f_{j}\right)$ for all $i \neq j$, and $n_{i}$ is a positive integer.

(c) Let $K^{i}$ be the $\mathbb{Z}$-graded total quotient ring of $R^{i}=k[x, y] /\left(f_{i}^{n_{i}}\right)$ for $1 \leqslant$ $i \leqslant m$. Then $K_{\geqslant 0} \simeq K_{\geqslant 0}^{1} \times \cdots \times K_{\geqslant 0}^{m}$ holds and $K_{\geqslant 0}^{i}$ is indecomposable in $\mathrm{CM}^{\mathbb{Z}} R$.

(d) Let $\left(\alpha_{i}^{\prime}: \beta_{i}^{\prime}\right) \in \mathbb{P}_{k}^{1}$ be a point different from $\left(\alpha_{i}: \beta_{i}\right)$. Then $\Gamma$ is presented by the quiver

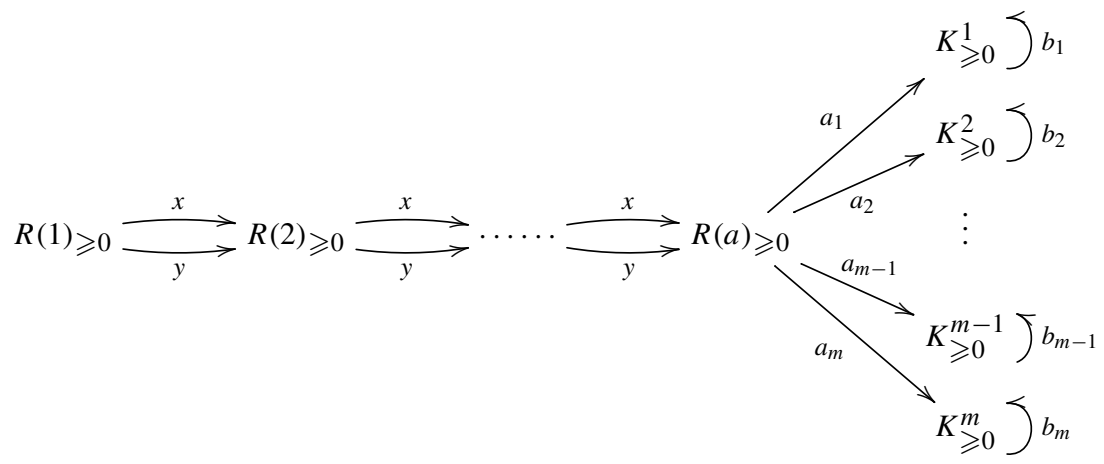

with relations

$$
x y=y x, \quad b_{i}^{n_{i}}=0, \quad a_{i}\left(\alpha_{i} x+\beta_{i} y\right)=b_{i} a_{i}\left(\alpha_{i}^{\prime} x+\beta_{i}^{\prime} y\right) .
$$

(e) $n_{1}=\cdots=n_{m}=1$ holds if and only if gl.dim $\Gamma<\infty$ if and only if gl.dim $\Gamma \leqslant 2$.

In (e), one can show that $\Gamma$ is derived equivalent to $k \times k$ if $n=2$, a path algebra of type $D_{4}$ if $n=3$, and a canonical algebra of type $(2,2,2,2)$ of $n=4$ (see [30] and Proposition 2.4(a)). Also note that if $n \geqslant 4$, then $\Gamma$ is not derived equivalent to a hereditary $k$-algebra by (b) above.

2.2. Simple curve singularities. In this subsection, we study simple curve singularities. They are precisely the ADE singularities when the base field is algebraically closed and the characteristic is different from 2, 3 and 5 [54, Section 9]. Our result is the following.

THEOREM 2.2. Let $R=k[x, y] /(f)$ be an ADE singularity over an arbitrary field $k$ with minimal grading given by the list below. Then $\mathrm{CM}^{\mathbb{Z}} R$ is triangle equivalent to $\mathrm{D}^{\mathrm{b}}(\bmod k Q)$, where $Q$ is a Dynkin quiver of the following type. 


\begin{tabular}{|c||c|c|c|c|c|}
\hline$R$ & $A_{n}$ & $D_{n}$ & $E_{6}$ & $E_{7}$ & $E_{8}$ \\
\hline$f$ & $x^{n+1}-y^{2}$ & $x^{n-1}-x y^{2}$ & $x^{4}-y^{3}$ & $x^{3} y-y^{3}$ & $x^{5}-y^{3}$ \\
\hline \multirow{2}{*}{$(\operatorname{deg} x, \operatorname{deg} y)$} & $\begin{array}{c}(2, n-2) n \text { is odd } \\
\left(1, \frac{n+1}{2}\right) n \text { is odd } \\
(2, n+1) n \text { is even }\end{array}$ & $\begin{array}{c}\left(1, \frac{n}{2}-1\right) n \text { is even } \\
(3,4)\end{array}$ & $(2,3)$ & $(3,5)$ \\
\hline$Q$ & $\begin{array}{c}D_{\frac{n+3}{2}} n \text { is odd } \\
A_{n} n \text { is even }\end{array}$ & $\begin{array}{c}A_{2 n-3} n \text { is odd } \\
D_{n} n \text { is even }\end{array}$ & $E_{6}$ & $E_{7}$ & $E_{8}$ \\
\hline
\end{tabular}

This is an analogue of (1.2) in dimension 2. The difference of types of $R$ and $Q$ was observed in [17] (see also [54, 72]). We will prove Theorem 2.2 in Section 4.7.

Our Theorem 2.2 immediately recovers the following well-known results.

COROLlary 2.3. Let $R=k[x, y] /(f)$ be as in Theorem 2.2, and $\widehat{R}$ the completion of $R$ at $R_{>0}$.

(a) [4] There are only finitely many indecomposable objects in $\mathrm{CM}^{\mathbb{Z}} R$ up to isomorphisms and degree shift. The stable Auslander-Reiten quiver of $\mathrm{CM}^{\mathbb{Z}} R$ is $\mathbb{Z} Q$ (see $[28]$ ).

(b) $[19,27,42]$ There are only finitely many indecomposable objects in $\mathrm{CM} \widehat{R}$ up to isomorphisms.

Proof. (a) is immediate from Theorem 2.2. (b) follows from (a) and [7, Theorem 5].

2.3. Curve singularities $\boldsymbol{T}_{p q}$. Drozd-Greuel classified commutative noetherian rings in dimension one, which are CM-tame in terms of $T_{p q}$ singularities [18]. Recall that $T_{p q}$ singularities over an algebraically closed field $k$ whose characteristic is different from 2 have the form $k[x, y] /(f)$, where $f=x^{p}+\lambda x^{2} y^{2}+y^{q}$ with $p \leqslant q$ and $\lambda \in k \backslash\{0,1\}$.

In this subsection, we deal with $\mathbb{Z}$-graded $T_{p q}$ singularities such that the variables $x$ and $y$ are homogeneous. This is precisely the case when $(p, q, \operatorname{deg} x$, $\operatorname{deg} y)$ is either $(4,4,1,1)$ or $(3,6,2,1)$. Our result below covers a slightly more general class of rings. Recall that a canonical algebra of type $(2,2,2,2)$ is given by the following quiver with relations for $\lambda \in k \backslash\{0,1\}[65]$.

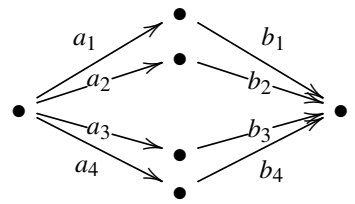

$$
\begin{aligned}
b_{1} a_{1}+b_{2} a_{2}+b_{3} a_{3} & =0 \\
b_{1} a_{1}+\lambda b_{2} a_{2}+b_{4} a_{4} & =0 .
\end{aligned}
$$


This algebra is derived equivalent to the weighted projective line of type $(2,2,2,2)[22]$.

We will prove the following result in Section 4.8.

Proposition 2.4. Let $k$ be an arbitrary field and $R=k[x, y] /(f)$, where

(a) $f=\prod_{i=1}^{4}\left(x-\alpha_{i} y\right)$ and $(\operatorname{deg} x, \operatorname{deg} y)=(1,1)$; or

(b) $f=\prod_{i=1}^{3}\left(x-\alpha_{i} y^{2}\right)$ and $(\operatorname{deg} x, \operatorname{deg} y)=(2,1)$.

If $R$ is reduced, then $\underline{\mathrm{CM}}^{\mathbb{Z}} R$ is triangle equivalent to $\mathrm{D}^{\mathrm{b}}(\bmod C)$, where $C$ is a canonical algebra of type $(2,2,2,2)$ with $\lambda=\left(\alpha_{1}-\alpha_{4}\right)\left(\alpha_{2}-\alpha_{3}\right)\left(\alpha_{1}-\alpha_{3}\right)^{-1}\left(\alpha_{2}-\right.$ $\left.\alpha_{4}\right)^{-1}$ for $(a)$ and $\lambda=\left(\alpha_{2}-\alpha_{3}\right)\left(\alpha_{1}-\alpha_{3}\right)^{-1}$ for $(b)$.

Consequently, $\underline{\mathrm{CM}}^{\mathbb{Z}} R$ is triangle equivalent to $\mathrm{D}^{\mathrm{b}}(\operatorname{coh} \mathbb{X})$, where $\mathbb{X}$ is the weighted projective line of type $(2,2,2,2)$. It will be interesting to find out a direct explanation of this equivalence.

2.4. Nonreduced examples. In this subsection, let $k$ be an arbitrary field and

$$
R=k[x, y] /\left(y^{2}\right) \quad \text { with } \operatorname{deg} x=n \geqslant 1 \text { and } \operatorname{deg} y=1 .
$$

When $n \geqslant 2$, this gives a typical example of rings with negative $a$-invariant. It is known as a Bass order in a non-semisimple algebra [34] and as a CM-countable ring $[12,54]$.

PROPOSITION 2.5. Under the above setting, the following holds true.

(a) The a-invariant of $R$ is $1-n$, and we have $K(n) \simeq K$.

(b) $\operatorname{per}(\mathrm{qgr} R)$ is triangle equivalent to $\mathrm{K}^{\mathrm{b}}(\operatorname{proj} \Lambda)$ for $\Lambda:=k Q /\left(z^{2}\right)$, where $Q$ is the following quiver with $Q_{0}=\mathbb{Z} / n \mathbb{Z}$.

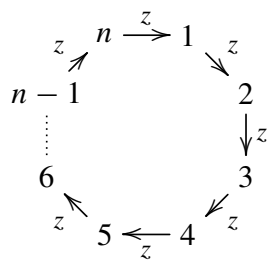

(c) The Auslander-Reiten quiver of $\operatorname{per}(\mathrm{qgr} R) \simeq \mathrm{K}^{\mathrm{b}}(\operatorname{proj} \Lambda)$ has $n$ connected components. For $i \in \mathbb{Z} / n \mathbb{Z}$, let $P^{i}=e_{i} \Lambda$ for the idempotent $e_{i} \in \Lambda$ 
corresponding to the vertex $i$, and for $a, b \in \mathbb{Z}$ with $b \geqslant 0$, let $X_{a, b}^{i}$ be the complex

$$
\cdots \rightarrow 0 \rightarrow P^{i} \stackrel{z}{\rightarrow} P^{i+1} \stackrel{z}{\rightarrow} P^{i+2} \stackrel{z}{\rightarrow} \cdots \stackrel{z}{\rightarrow} P^{i+b-1} \stackrel{z}{\rightarrow} P^{i+b} \rightarrow 0 \rightarrow \cdots
$$

whose nonzero degrees are $a, a+1, \ldots, a+b$. Then the following is $a$ connected component for $i \in \mathbb{Z} / n \mathbb{Z}$.

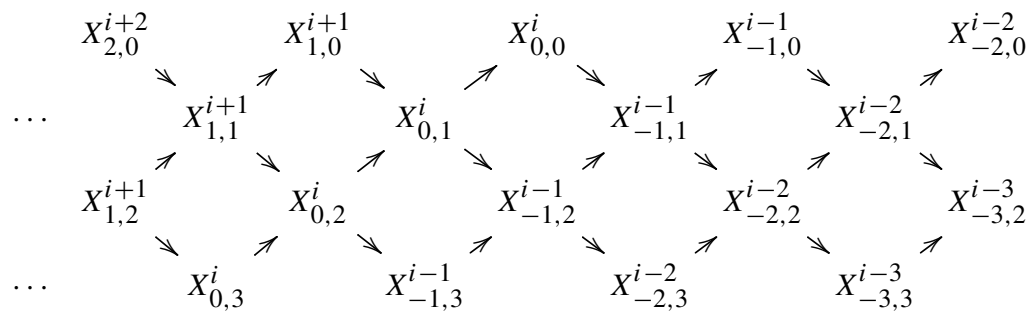

(d) ${\underline{\mathrm{MM}_{0}^{\mathbb{Z}}}}_{0}^{\mathbb{Z}}$ is triangle equivalent to $\mathrm{K}^{\mathrm{b}}(\operatorname{proj} \Lambda) /$ thick $P$, where $P=\bigoplus_{i=1}^{n-1} P^{i}$.

(e) $\underline{\mathrm{CM}}_{0}^{\mathbb{Z}} R$ has a silting object $R(1)_{\geqslant 0}$, and has a tilting object if and only if $n=1$. It is triangle equivalent to the perfect derived category per $k[w] /\left(w^{2}\right)$ for the differential graded $(D G)$ algebra $k[w] /\left(w^{2}\right)$ with $\operatorname{deg} w=1-n$ and zero differential.

(f) The Auslander-Reiten quiver of $\underline{\mathrm{CM}}_{0}^{\mathbb{Z}} R$ has $n$ connected components. For $i>0$ and $j \in \mathbb{Z}$, let $R^{i}:=R+\left\langle x^{-\ell} y \mid 1 \leqslant \ell \leqslant i\right\rangle_{k}$ and $R^{i, j}=R^{i}(j)$. Then the following is a connected component for $j \in \mathbb{Z} / n \mathbb{Z}$.

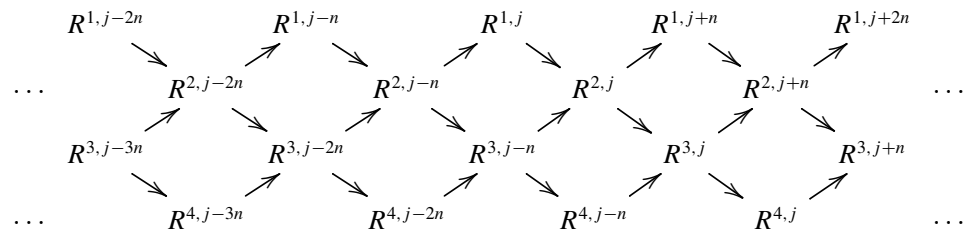

We will give a proof of Proposition 2.5 in Section 4.9. Note that the AuslanderReiten quivers of per(qgr $R)$ and $\underline{\mathrm{CM}}_{0}^{\mathbb{Z}} R$ are isomorphic, but they are not triangle equivalent. 


\section{Realizing Verdier quotients as thick subcategories}

Throughout this subsection, we assume that $A$ is a $\mathbb{Z}$-graded IwanagaGorenstein ring, that is,

- $A$ is a noetherian ring on each side with inj.dim $A_{A}<\infty$ and inj. $\operatorname{dim}_{A} A<\infty$.

We denote by $\bmod ^{\mathbb{Z}} A$ the category of $\mathbb{Z}$-graded finitely generated (right) $A$-modules, by proj $^{\mathbb{Z}} A$ the category of $\mathbb{Z}$-graded finitely generated projective $A$-modules, and by $\bmod _{0}^{\mathbb{Z}} A$ the category of $\mathbb{Z}$-graded $A$-modules of finite length.

Under certain conditions, it is known $[41,62]$ that two Verdier quotients $\mathrm{D}^{\mathrm{b}}\left(\bmod ^{\mathbb{Z}} A\right) / \mathrm{K}^{\mathrm{b}}\left(\operatorname{proj}^{\mathbb{Z}} A\right)$ and $\mathrm{D}^{\mathrm{b}}\left(\bmod ^{\mathbb{Z}} A\right) / \mathrm{D}^{\mathrm{b}}\left(\bmod _{0}^{\mathbb{Z}} A\right)$ can be realized as thick subcategories of $\mathrm{D}^{\mathrm{b}}\left(\bmod ^{\mathbb{Z}} A\right)$. The aim of this section is to give an analogous result for the thick subcategory

$$
\mathcal{D}_{A}:=\operatorname{thick}\left\{\operatorname{proj}^{\mathbb{Z}} A, \bmod _{0}^{\mathbb{Z}} A\right\} \subseteq \mathrm{D}^{\mathrm{b}}\left(\bmod ^{\mathbb{Z}} A\right),
$$

and its Verdier quotients $\mathcal{D}_{A} / \mathrm{K}^{\mathrm{b}}\left(\operatorname{proj}^{\mathbb{Z}} A\right)$ and $\mathcal{D}_{A} / \mathrm{D}^{\mathrm{b}}\left(\bmod _{0}^{\mathbb{Z}} A\right)$.

For a subset $I$ of $\mathbb{Z}$, let $\bmod ^{I} A$ be the full subcategory of $\bmod ^{\mathbb{Z}} A$ consisting of all $X$ satisfying $X_{i}=0$ for all $i \in \mathbb{Z} \backslash I$. For an integer $\ell \in \mathbb{Z}$, let $\bmod ^{\geqslant \ell} A:=$ $\bmod ^{[\ell, \infty)} A, \bmod ^{\leqslant \ell} A:=\bmod ^{(-\infty, \ell]} A$ and so on. Then $\mathrm{D}^{\mathrm{b}}\left(\bmod ^{\geqslant \ell} A\right)$ can be regarded as a thick subcategory of $D^{\mathrm{b}}\left(\bmod ^{\mathbb{Z}} A\right)$. Let

$$
\mathcal{D}_{A}^{\geqslant \ell}=\mathcal{D}_{A}^{>\ell-1}:=\mathcal{D}_{A} \cap D^{\mathrm{b}}\left(\bmod ^{\geqslant \ell} A\right) .
$$

Let $\bmod _{0}^{\geqslant \ell} A:=\bmod ^{\geqslant \ell} A \cap \bmod _{0}^{\mathbb{Z}} A, \bmod _{0}^{\leqslant \ell} A:=\bmod ^{\leqslant \ell} A \cap \bmod _{0}^{\mathbb{Z}} A=$ $\bmod ^{\leqslant \ell} A$ and so on. Similarly, let $\operatorname{proj}^{I} A$ be the full subcategory of $\operatorname{proj}^{\mathbb{Z}} A$ consisting of all $P$, which are generated by homogeneous elements of degrees in $I$. Let $\operatorname{proj}^{\geqslant \ell} A:=\operatorname{proj}^{[\ell, \infty)} A, \operatorname{proj}^{\leqslant \ell} A:=\operatorname{proj}^{(-\infty, \ell]} A$ and so on.

Since $A$ is Iwanaga-Gorenstein, we have a duality [57, Corollary 2.11]

$$
(-)^{*}:=\mathbf{R H o m}_{A}(-, A): \mathrm{D}^{\mathrm{b}}\left(\bmod ^{\mathbb{Z}} A\right) \simeq \mathrm{D}^{\mathrm{b}}\left(\bmod ^{\mathbb{Z}} A^{\mathrm{op}}\right) .
$$

We consider the following three conditions.

(A1) $A=\bigoplus_{i \geqslant 0} A_{i}$ and gl.dim $A_{0}<\infty$.

(A2) $A_{0}$ is an artinian ring.

(A3) There exists $a \in \mathbb{Z}$ such that $(-)^{*}$ restricts to a duality $(-)^{*}: \mathrm{D}^{\mathrm{b}}\left(\bmod ^{0} A\right) \simeq$ $\mathrm{D}^{\mathrm{b}}\left(\bmod ^{a} A^{\mathrm{op}}\right)$. 
For example, our $R$ satisfying (R1) and (R2) satisfies these conditions for the $a$-invariant $a$ of $R$.

Let $\mathcal{X}$ and $\mathcal{Y}$ be full subcategories in a triangulated category $\mathcal{T}$. We denote by $\mathcal{X} * \mathcal{Y}$ the full subcategory of $\mathcal{T}$ whose objects consist of $Z \in \mathcal{T}$ such that there is a triangle $X \rightarrow Z \rightarrow Y \rightarrow X[1]$ with $X \in \mathcal{X}$ and $Y \in \mathcal{Y}$. When $\operatorname{Hom}_{\mathcal{T}}(\mathcal{X}, \mathcal{Y})=0$ holds, we write $\mathcal{X} * \mathcal{Y}=\mathcal{X} \perp \mathcal{Y}$. For full subcategories $\mathcal{X}_{1}, \ldots, \mathcal{X}_{n}$, we define $\mathcal{X}_{1} * \cdots * \mathcal{X}_{n}$ and $\mathcal{X}_{1} \perp \cdots \perp \mathcal{X}_{n}$ inductively.

We are ready to state the following main result in this section.

THEOREM 3.1. Let $A$ be a $\mathbb{Z}$-graded Iwanaga-Gorenstein ring satisfying $A=$ $A_{\geqslant 0}$, and $\ell$ an integer.

(a) If condition (A1) is satisfied, then we have a semiorthogonal decomposition

$$
\mathcal{D}_{A}=\mathrm{K}^{\mathrm{b}}\left(\operatorname{proj}^{<\ell} A\right) \perp\left(\mathcal{D}_{A}^{\geqslant \ell} \cap\left(\mathcal{D}_{A^{\text {op }}}^{>-\ell}\right)^{*}\right) \perp \mathrm{K}^{\mathrm{b}}\left(\text { proj }^{\geqslant \ell} A\right) .
$$

The natural functor $\mathcal{D}_{A} \rightarrow \mathcal{D}_{A} / \mathrm{K}^{\mathrm{b}}\left(\mathrm{proj}^{\mathbb{Z}} A\right)$ restricts to a triangle equivalence

$$
\mathcal{D}_{A}^{\geqslant \ell} \cap\left(\mathcal{D}_{A^{\text {op }}}^{>-\ell}\right)^{*} \simeq \mathcal{D}_{A} / \mathrm{K}^{\mathrm{b}}\left(\operatorname{proj}^{\mathbb{Z}} A\right)
$$

(b) If conditions (A2) and (A3) are satisfied, then we have a semiorthogonal decomposition

$$
\mathcal{D}_{A}=\mathrm{D}^{\mathrm{b}}\left(\bmod _{0}^{\geqslant \ell} A\right) \perp\left(\mathcal{D}_{A}^{\geqslant \ell} \cap\left(\mathcal{D}_{A^{\text {op }}}^{>a-\ell}\right)^{*}\right) \perp \mathrm{D}^{\mathrm{b}}\left(\bmod _{0}^{<\ell} A\right) .
$$

The natural functor $\mathcal{D}_{A} \rightarrow \mathcal{D}_{A} / \mathrm{D}^{\mathrm{b}}\left(\bmod _{0}^{\mathbb{Z}} A\right)$ restricts to a triangle equivalence

$$
\mathcal{D}_{A}^{\geqslant \ell} \cap\left(\mathcal{D}_{A^{\text {op }}}^{>a-\ell}\right)^{*} \simeq \mathcal{D}_{A} / D^{\mathrm{b}}\left(\bmod _{0}^{\mathbb{Z}} A\right)
$$

(c) Assume that conditions (A1), (A2) and (A3) are satisfied. If $a \geqslant 0$, then we have a semiorthogonal decomposition

$$
\mathcal{D}_{A}^{\geqslant \ell-a} \cap\left(\mathcal{D}_{A^{\circ \mathrm{op}}}^{>a-\ell}\right)^{*}=\left(\mathcal{D}_{A}^{\geqslant \ell} \cap\left(\mathcal{D}_{A^{\text {op }}}^{>a-\ell}\right)^{*}\right) \perp \mathrm{D}^{\mathrm{b}}\left(\bmod ^{[\ell-a, \ell-1]} A\right) .
$$

If $a \leqslant 0$, then we have a semiorthogonal decomposition

$$
\mathcal{D}_{A}^{\geqslant \ell} \cap\left(\mathcal{D}_{A^{\text {op }}}^{>a-\ell}\right)^{*}=\mathrm{K}^{\mathrm{b}}\left(\operatorname{proj}^{[\ell, \ell-a-1]} A\right) \perp\left(\mathcal{D}_{A}^{\geqslant \ell-a} \cap\left(\mathcal{D}_{A^{\text {op }}}^{>a-\ell}\right)^{*}\right) .
$$

Immediately, we obtain the following analogue of Orlov's semiorthogonal decompositions [62]. 
Corollary 3.2. Assume that (A1), (A2) and (A3) are satisfied. For $\ell \in \mathbb{Z}$, there exist fully faithful triangle functors $F_{\ell}: \mathcal{D}_{A} / \mathrm{K}^{\mathrm{b}}\left(\mathrm{proj}^{\mathbb{Z}} A\right) \rightarrow \mathcal{D}_{A}$ and $G_{\ell}$ : $\mathcal{D}_{A} / \mathrm{D}^{\mathrm{b}}\left(\bmod _{0}^{\mathbb{Z}} A\right) \rightarrow \mathcal{D}_{A}$ and a semiorthogonal decomposition

$$
\begin{array}{ll}
G_{\ell}\left(\mathcal{D}_{A} / \mathrm{D}^{\mathrm{b}}\left(\bmod _{0}^{\mathbb{Z}} A\right)\right) \simeq \mathrm{K}^{\mathrm{b}}\left(\operatorname{proj}^{[\ell, \ell-a-1]} A\right) \perp F_{\ell}\left(\mathcal{D}_{A} / \mathrm{K}^{\mathrm{b}}\left(\operatorname{proj}^{\mathbb{Z}} A\right)\right) & \text { if } a<0, \\
F_{\ell}\left(\mathcal{D}_{A} / \mathrm{K}^{\mathrm{b}}\left(\operatorname{proj}^{\mathbb{Z}} A\right)\right) \simeq G_{\ell}\left(\mathcal{D}_{A} / \mathrm{D}^{\mathrm{b}}\left(\bmod _{0}^{\mathbb{Z}} A\right)\right) & \text { if } a=0, \\
F_{\ell}\left(\mathcal{D}_{A} / \mathrm{K}^{\mathrm{b}}\left(\operatorname{proj}^{\mathbb{Z}} A\right)\right) \simeq G_{\ell}\left(\mathcal{D}_{A} / \mathrm{D}^{\mathrm{b}}\left(\bmod _{0}^{\mathbb{Z}} A\right)\right) \perp \mathrm{D}^{\mathrm{b}}\left(\bmod ^{[\ell-a, \ell-1]} A\right) & \text { if } a>0 .
\end{array}
$$

We refer to [56] for analogous results to Theorem 3.1.

The rest of this section is devoted to proving Theorem 3.1 and Corollary 3.2. We start with the following easy observation.

LEMMA 3.3. Let $\mathcal{T}$ be a triangulated category and $\mathcal{T}=\mathcal{X} \perp \mathcal{Y}$ a semiorthogonal decomposition.

(a) If $\mathcal{Z}$ is a thick subcategory of $\mathcal{T}$ such that $\mathcal{X} \subseteq \mathcal{Z}$, then $\mathcal{Z}=\mathcal{X} \perp(\mathcal{Y} \cap \mathcal{Z})$.

(b) If $\mathcal{Z}$ is a thick subcategory of $\mathcal{T}$ such that $\mathcal{Y} \subseteq \mathcal{Z}$, then $\mathcal{Z}=(\mathcal{X} \cap \mathcal{Z}) \perp \mathcal{Y}$.

(c) If $\mathcal{T}=\mathcal{X}^{\prime} \perp \mathcal{Y}^{\prime}$ is a semiorthogonal decomposition such that $\mathcal{X} \subseteq \mathcal{X}^{\prime}$ (or equivalently, $\mathcal{Y} \supseteq \mathcal{Y}^{\prime}$ ), then we have a semiorthogonal decomposition $\mathcal{T}=\mathcal{X} \perp\left(\mathcal{Y} \cap \mathcal{X}^{\prime}\right) \perp \mathcal{Y}^{\prime}$

Proof. (a) and (b) are easy. By (a), we have $\mathcal{X}^{\prime}=\mathcal{X} \perp\left(\mathcal{Y} \cap \mathcal{X}^{\prime}\right)$ and hence $\mathcal{T}=\mathcal{X}^{\prime} \perp \mathcal{Y}^{\prime}=\mathcal{X} \perp\left(\mathcal{Y} \cap \mathcal{X}^{\prime}\right) \perp \mathcal{Y}^{\prime}$

We need the following elementary observation (for example, $[62,2.3]$ ).

Proposition 3.4. Let $A$ be a $\mathbb{Z}$-graded Iwanaga-Gorenstein ring satisfying (A1). Then there exists a semiorthogonal decomposition $\mathrm{K}\left(\operatorname{proj}^{\mathbb{Z}} A\right)=$ $\mathrm{K}\left(\right.$ proj $\left.^{<\ell} A\right) \perp \mathrm{K}\left(\right.$ proj $\left.^{\geqslant \ell} A\right)$, which gives $\mathrm{K}^{\mathrm{b}}\left(\operatorname{proj}^{\mathbb{Z}} A\right)=\mathrm{K}^{\mathrm{b}}\left(\operatorname{proj}^{<\ell} A\right) \perp$ $\mathrm{K}^{\mathrm{b}}\left(\operatorname{proj}^{\geqslant \ell} A\right)$ and $\mathrm{D}^{\mathrm{b}}\left(\bmod ^{\mathbb{Z}} A\right)=\mathrm{K}^{\mathrm{b}}\left(\operatorname{proj}^{<\ell} A\right) \perp \mathrm{D}^{\mathrm{b}}\left(\bmod ^{\geqslant \ell} A\right)$.

Now we prove Theorem 3.1(a).

Proof of Theorem 3.1 (a). We have $\mathrm{D}^{\mathrm{b}}\left(\bmod ^{\mathbb{Z}} A\right)=\mathrm{K}^{\mathrm{b}}\left(\operatorname{proj}^{<\ell} A\right) \perp \mathrm{D}^{\mathrm{b}}\left(\bmod ^{\geqslant \ell} A\right)$ by Proposition 3.4. Applying Lemma 3.3(a) to $\mathcal{X}:=\mathrm{K}^{\mathrm{b}}\left(\right.$ proj $\left.^{<\ell} A\right) \subseteq \mathcal{Z}:=\mathcal{D}_{A}$, we have

$$
\mathcal{D}_{A}=\mathrm{K}^{\mathrm{b}}\left(\operatorname{proj}^{<\ell} A\right) \perp\left(\mathcal{D}_{A} \cap \mathrm{D}^{\mathrm{b}}\left(\bmod ^{\geqslant \ell} A\right)\right)=\mathrm{K}^{\mathrm{b}}\left(\operatorname{proj}^{<\ell} A\right) \perp \mathcal{D}_{A}^{\geqslant \ell} .
$$


Replacing $\ell$ by $1-\ell$, we have $\mathcal{D}_{A^{\text {op }}}=\mathrm{K}^{\mathrm{b}}\left(\operatorname{proj}^{\leqslant-\ell} A^{\text {op }}\right) \perp \mathcal{D}_{A^{\text {op }}}^{>-\ell}$. Applying $(-)^{*}$, we have

$$
\mathcal{D}_{A}=\left(\mathcal{D}_{A^{\text {op }}}^{>-\ell}\right)^{*} \perp \mathrm{K}^{\mathrm{b}}\left(\operatorname{proj}^{\leqslant-\ell} A^{\mathrm{op}}\right)^{*}=\left(\mathcal{D}_{A^{\mathrm{op}}}^{>-\ell}\right)^{*} \perp \mathrm{K}^{\mathrm{b}}\left(\text { proj }^{\geqslant \ell} A\right) .
$$

Since $\mathcal{D}_{A}^{\geqslant \ell} \supseteq \mathrm{K}^{\mathrm{b}}$ (proj ${ }^{\geqslant \ell} A$ ), applying Lemma 3.3(c) to (3.1) and (3.2) gives $\mathcal{D}_{A}=$ $\mathrm{K}^{\mathrm{b}}\left(\right.$ proj $\left.^{<\ell} A\right) \perp\left(\mathcal{D}_{A}^{\geqslant \ell} \cap\left(\mathcal{D}_{A^{\text {op }}}^{>-\ell}\right)^{*}\right) \perp \mathrm{K}^{\mathrm{b}}\left(\right.$ proj $\left.^{\geqslant \ell} A\right)$ as desired. The last assertion follows from

$$
\mathcal{D}_{A} / \mathrm{K}^{\mathrm{b}}\left(\text { proj }^{\mathbb{Z}} A\right) \stackrel{\text { Prop. }}{=}{ }^{3.4} \mathcal{D}_{A} /\left(\mathrm{K}^{\mathrm{b}}\left(\operatorname{proj}^{<\ell} A\right) \perp \mathrm{K}^{\mathrm{b}}\left(\text { proj }^{\geqslant \ell} A\right)\right) \simeq \mathcal{D}_{A}^{\geqslant \ell} \cap\left(\mathcal{D}_{A^{\text {op }}}^{>-\ell}\right)^{*} \text {. }
$$

We also need the following elementary observation (for example, [62, 2.3]).

Proposition 3.5. Let $A$ be a $\mathbb{Z}$-graded Iwanaga-Gorenstein ring satisfying $A=A_{\geqslant 0}$ and (A2). Then there exist semiorthogonal decompositions $\mathrm{D}^{\mathrm{b}}\left(\bmod _{0}^{\mathbb{Z}} A\right)$ $=\mathrm{D}^{\mathrm{b}}\left(\bmod _{0}^{\geqslant \ell} A\right) \perp \mathrm{D}^{\mathrm{b}}\left(\bmod _{0}^{<\ell} A\right)$ and $\mathrm{D}^{\mathrm{b}}\left(\bmod ^{\mathbb{Z}} A\right)=\mathrm{D}^{\mathrm{b}}\left(\bmod ^{\geqslant \ell} A\right) \perp \mathrm{D}^{\mathrm{b}}$ $\left(\bmod _{0}^{<\ell} A\right)$.

Now we prove Theorem 3.1(b) and (c).

Proof of Theorem 3.1(b)(c). We have $\mathrm{D}^{\mathrm{b}}\left(\bmod ^{\mathbb{Z}} A\right)=\mathrm{D}^{\mathrm{b}}\left(\bmod ^{\geqslant \ell} A\right) \perp$ $\mathrm{D}^{\mathrm{b}}\left(\bmod _{0}^{<\ell} A\right)$ by Proposition 3.5. Applying Lemma 3.3(b) to $\mathcal{Y}:=$ $\mathrm{D}^{\mathrm{b}}\left(\bmod _{0}^{<\ell} A\right) \subseteq \mathcal{Z}:=\mathcal{D}_{A}$, we have

$$
\mathcal{D}_{A}=\left(\mathcal{D}_{A} \cap \mathrm{D}^{\mathrm{b}}\left(\bmod ^{\geqslant \ell} A\right)\right) \perp \mathrm{D}^{\mathrm{b}}\left(\bmod _{0}^{<\ell} A\right)=\mathcal{D}_{A}^{\geqslant \ell} \perp \mathrm{D}^{\mathrm{b}}\left(\bmod _{0}^{<\ell} A\right) .
$$

Replacing $\ell$ by $a-\ell+1$, we have $\mathcal{D}_{A^{\text {op }}}=\mathcal{D}_{A^{\text {op }}}^{>a-\ell} \perp \mathrm{D}^{\mathrm{b}}\left(\bmod _{0}^{\leqslant a-\ell} A^{\text {op }}\right)$. Applying $(-)^{*}$, we have

$$
\mathcal{D}_{A}=\mathrm{D}^{\mathrm{b}}\left(\bmod _{0}^{\leqslant a-\ell} A^{\mathrm{op}}\right)^{*} \perp\left(\mathcal{D}_{A^{\mathrm{op}}}^{>a-\ell}\right)^{*} \stackrel{(\mathrm{A} 3)}{=} \mathrm{D}^{\mathrm{b}}\left(\bmod _{0}^{\geqslant \ell} A\right) \perp\left(\mathcal{D}_{A^{\mathrm{op}}}^{>a-\ell}\right)^{*} .
$$

Since $\mathcal{D}_{A}^{\geqslant \ell} \supseteq D^{\mathrm{b}}\left(\bmod _{0}^{\geqslant \ell} A\right)$, applying Lemma 3.3(c) to (3.3) and (3.4) gives $\mathcal{D}_{A}=$ $\mathrm{D}^{\mathrm{b}}\left(\bmod _{0}^{\geqslant \ell} A\right) \perp\left(\mathcal{D}_{A}^{\geqslant \ell} \cap\left(\mathcal{D}_{A^{\text {op }}}^{>a-\ell}\right)^{*}\right) \perp \mathrm{D}^{\mathrm{b}}\left(\bmod _{0}^{<\ell} A\right)$ as desired. The last assertion follows from

$\mathcal{D}_{A} / \mathrm{D}^{\mathrm{b}}\left(\bmod _{0}^{\mathbb{Z}} A\right) \stackrel{\text { Prop. } 3.5}{=} \mathcal{D}_{A} /\left(\mathrm{D}^{\mathrm{b}}\left(\bmod _{0}^{\geqslant \ell} A\right) \perp \mathrm{D}^{\mathrm{b}}\left(\bmod _{0}^{<\ell} A\right)\right) \simeq \mathcal{D}_{A}^{\geqslant \ell} \cap\left(\mathcal{D}_{A^{\text {op }}}^{>a-\ell}\right)^{*}$.

(c) Assume $a \geqslant 0$. Then $\mathcal{D}_{A}^{\geqslant \ell-a}=\mathcal{D}_{A}^{\geqslant \ell} \perp \mathrm{D}^{\mathrm{b}}\left(\bmod ^{[\ell-a, \ell-1]} A\right)$ holds. Since $\mathrm{D}^{\mathrm{b}}\left(\bmod ^{[\ell-a, \ell-1]} A\right)=\mathrm{D}^{\mathrm{b}}\left(\bmod ^{[a+1-\ell, 2 a-\ell]} A^{\mathrm{op}}\right)^{*} \subseteq\left(\mathcal{D}_{A^{\mathrm{op}}}^{>a-\ell}\right)^{*}$, the assertion follows from Lemma 3.3(b).

Assume $a \leqslant 0$. Then $\mathcal{D}_{A}^{\geqslant \ell}=\mathrm{K}^{\mathrm{b}}\left(\right.$ proj $\left.^{[\ell, \ell-a-1]} A\right) \perp \mathcal{D}_{A}^{\geqslant \ell-a}$ holds. Since $\mathrm{K}^{\mathrm{b}}\left(\operatorname{proj}^{[\ell, \ell-a-1]} A\right)=\mathrm{K}^{\mathrm{b}}\left(\operatorname{proj}^{[a+1-\ell,-\ell]} A^{\text {op }}\right)^{*} \subseteq\left(\mathcal{D}_{A^{\mathrm{op}}}^{>a-\ell}\right)^{*}$, the assertion follows from Lemma 3.3(a). 


\section{Proof of our results}

4.1. Preliminaries. We start with recalling the central notion of tilting objects.

DEFINITION 4.1. Let $\mathcal{T}$ be a triangulated category with suspension functor [1]. A full subcategory of $\mathcal{T}$ is thick if it is closed under cones, $[ \pm 1]$ and direct summands. We call an object $T \in \mathcal{T}$ tilting (respectively, silting) if $\operatorname{Hom}_{\mathcal{T}}(T, T[i])=0$ holds for all integers $i \neq 0$ (respectively, $i>0$ ), and the smallest thick subcategory of $\mathcal{T}$ containing $T$ is $\mathcal{T}$.

The principal example of tilting objects appears in $\mathrm{K}^{\mathrm{b}}(\operatorname{proj} \Lambda)$ for a ring $\Lambda$. It has a tilting object given by the stalk complex $\Lambda$ concentrated in degree zero, and a certain converse holds in the sense of Proposition 4.2. More generally, tilting objects in $\mathrm{K}^{\mathrm{b}}(\operatorname{proj} \Lambda)$ are precisely tilting complexes [64] of $\Lambda$, where tilting $\Lambda$-modules [28] are special examples. Note that there are some variations of the definitions of tilting objects $[3,48]$, for example, the last condition thick $T=\mathcal{T}$ is sometimes replaced by the condition 'if $X \in \mathcal{T}$ satisfies $\operatorname{Hom}_{\mathcal{T}}(T, X[i])=0$ for all $i \in \mathbb{Z}$, then $X=0$ '. If $\mathcal{T}$ has arbitrary coproducts, then $T$ is assumed to be compact and 'thick subcategory' in the last condition is replaced by 'localizing subcategory'.

Recall that a triangulated category is called algebraic if it is triangle equivalent to the stable category of a Frobenius category. Let us recall the following wellknown result due to Keller [47] (see [51] for a detailed proof).

PROPOSITION 4.2 [47]. Let $\mathcal{T}$ be an algebraic triangulated category with a tilting object $T$. There exists a triangle equivalence $F: \mathcal{T} \rightarrow \mathrm{K}^{\mathrm{b}}\left(\operatorname{proj}_{\operatorname{End}}(T)\right)$ up to direct summands such that $F(T)=\operatorname{End}_{\mathcal{T}}(T)$. It is a triangle equivalence if $\mathcal{T}$ is idempotent complete.

Let $k$ be a field and $D$ the $k$-dual. For a finite-dimensional $k$-algebra $\Lambda$, we denote by

$$
v=-\stackrel{\mathrm{L}}{\otimes}_{\Lambda}(D \Lambda): \mathrm{K}^{\mathrm{b}}(\operatorname{proj} \Lambda) \simeq \mathrm{K}^{\mathrm{b}}(\operatorname{inj} \Lambda)
$$

the Nakayama functor. If $\Lambda$ is Iwanaga-Gorenstein, then $v$ is an autoequivalence of $\mathrm{K}^{\mathrm{b}}(\operatorname{proj} \Lambda)=\mathrm{K}^{\mathrm{b}}(\operatorname{inj} \Lambda)$. The following result due to Happel is also well known.

Proposition 4.3 [28]. Let $\Lambda$ be a finite-dimensional $k$-algebra. Then we have a functorial isomorphism

$$
D \operatorname{Hom}_{\mathrm{D}(\operatorname{Mod} \Lambda)}(X, Y) \simeq \operatorname{Hom}_{\mathrm{D}(\operatorname{Mod} \Lambda)}(Y, v X)
$$


for any $X \in \mathrm{K}^{\mathrm{b}}(\operatorname{proj} \Lambda)$ and $Y \in \mathrm{D}(\operatorname{Mod} \Lambda)$. In particular, if $\Lambda$ is IwanagaGorenstein, then $\mathrm{K}^{\mathrm{b}}(\operatorname{proj} \Lambda)$ has a Serre functor $v$.

Now we prove the following general observation.

PROPOSITION 4.4. Let $\mathcal{T}$ be a Hom-finite $k$-linear algebraic triangulated category with Serre functor $\mathbb{S}$. Let $T \in \mathcal{T}$ be a tilting object and $\Lambda:=\operatorname{End}_{\mathcal{T}}(T)$. Then the following holds true.

(a) $\Lambda$ is an Iwanaga-Gorenstein k-algebra.

(b) There are a triangle equivalence $F: \mathcal{T} \simeq \mathrm{K}^{\mathrm{b}}$ (proj $\Lambda$ ) up to direct summands and the following commutative diagram up to an isomorphism of functors.

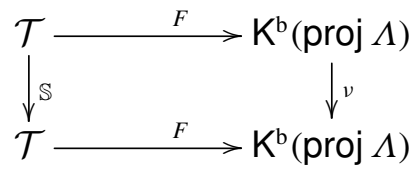

Proof. (a) By Proposition 4.2, we may regard $\mathcal{T}$ as a full triangulated subcategory of $\mathrm{K}^{\mathrm{b}}(\operatorname{proj} \Lambda)$ and $T=\Lambda$. Then we have isomorphisms

$$
\begin{aligned}
\operatorname{Hom}_{\mathcal{T}}(-, \mathbb{S} \Lambda) \simeq D \operatorname{Hom}_{\mathcal{T}}(\Lambda,-) & =\left.D \operatorname{Hom}_{\mathrm{D}(\operatorname{Mod} \Lambda)}(\Lambda,-)\right|_{\mathcal{T}} \\
& \left.\simeq \operatorname{Hom}_{\mathrm{D}(\operatorname{Mod} \Lambda)}(-, D \Lambda)\right|_{\mathcal{T}} .
\end{aligned}
$$

By Yoneda's lemma, there is a morphism $f: \mathbb{S} \Lambda \rightarrow D \Lambda$ in $\mathrm{D}(\operatorname{Mod} \Lambda)$, which induces an isomorphism $\left.\operatorname{Hom}_{\mathcal{T}}(-, \mathbb{S} \Lambda) \rightarrow \operatorname{Hom}_{\mathrm{D}(\operatorname{Mod} \Lambda)}(-, D \Lambda)\right|_{\mathcal{T}}$. Then the cone $C \in \mathrm{D}(\operatorname{Mod} \Lambda)$ of $f$ satisfies $\operatorname{Hom}_{\mathrm{D}(\operatorname{Mod} \Lambda)}(\mathcal{T}, C)=0$. Since $\Lambda \in \mathcal{T}$, we have $C=0$. Thus $D \Lambda \simeq \mathbb{S} \Lambda$ belongs to $\mathcal{T}$, and therefore proj.dim $(D \Lambda)_{\Lambda}<\infty$. On the other hand, since $\mathcal{T}^{\text {op }}$ also has a Serre functor, we have proj. $\operatorname{dim}_{\Lambda}(D \Lambda)<$ $\infty$. Thus $\Lambda$ is Iwanaga-Gorenstein.

(b) This is immediate from Proposition 4.3 and the uniqueness of Serre functors.

As an application of Proposition 4.4, we give a direct proof of the observation below. Note that it was known to experts as a consequence of [29, Theorem 3.4] and [63, Theorem I.2.4].

Proposition 4.5 [13, Corollary 3.9]. Let $\Lambda$ be a finite-dimensional k-algebra. Then $\mathrm{K}^{\mathrm{b}}(\operatorname{proj} \Lambda)$ has a Serre functor if and only if $\Lambda$ is Iwanaga-Gorenstein.

Proof. The 'if' part is Proposition 4.3, and the 'only if' part is Proposition 4.4. 
In the rest of this subsection, let $R$ be a $\mathbb{Z}$-graded Gorenstein ring in dimension $d$ such that $R=R_{\geqslant 0}$ and $k:=R_{0}$ is a field, and $a$ the $a$-invariant of $R$.

The following Auslander-Reiten-Serre duality is basic.

PROPOSITION 4.6 [6, 39]. Under the above setting, there is a functorial isomorphism

$$
\underline{\operatorname{Hom}}_{R}^{\mathbb{Z}}(X, Y) \simeq D \underline{\operatorname{Hom}}_{R}^{\mathbb{Z}}(Y, X(a)[d-1])
$$

for any $X \in \mathrm{CM}^{\mathbb{Z}} R$ and $Y \in \mathrm{CM}_{0}^{\mathbb{Z}} R$.

The results above give the following important observation.

THEOREM 4.7. Under the above setting, we assume that $\underline{\mathrm{CM}}_{0}^{\mathbb{Z}} R$ has a tilting object $U$.

(a) $\Lambda:=\underline{\operatorname{End}}_{R}^{\mathbb{Z}}(U)$ is an Iwanaga-Gorenstein ring.

(b) There are a triangle equivalence $F: \underline{\mathrm{CM}}_{0}^{\mathbb{Z}} R \simeq \mathrm{K}^{\mathrm{b}}$ (proj $\Lambda$ ) and the following commutative diagram up to an isomorphism of functors.

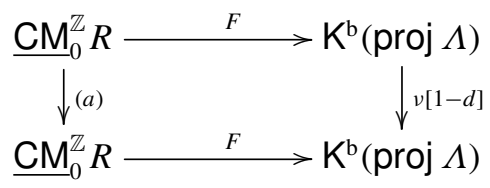

Proof. The assertion is immediate from Propositions 4.2, 4.4 and 4.6.

We give an analogue of Buchweitz's description of $\underline{\mathrm{CM}}^{\mathbb{Z}} R[11]$ for $\underline{\mathrm{CM}}_{0}^{\mathbb{Z}} R$.

Proposition 4.8. Under the above setting, let $\mathcal{D}_{R}:=\operatorname{thick}\{\operatorname{proj}\}^{\mathbb{Z}} R$, $\left.\bmod _{0}^{\mathbb{Z}} R\right\} \subseteq \mathrm{D}^{\mathrm{b}}\left(\bmod ^{\mathbb{Z}} R\right)$. Then the triangle equivalence $\mathrm{D}^{\mathrm{b}}\left(\bmod ^{\mathbb{Z}} R\right) / \mathrm{K}^{\mathrm{b}}$ $\left(\right.$ proj $\left.^{\mathbb{Z}} R\right) \simeq \underline{\mathrm{CM}}^{\mathbb{Z}} R$ restricts to a triangle equivalence

$$
\mathcal{D}_{R} / \mathrm{K}^{\mathrm{b}}\left(\operatorname{proj}^{\mathbb{Z}} R\right) \simeq \underline{\mathrm{CM}}_{0}^{\mathbb{Z}} R
$$

Proof. For any $\mathbb{Z}$-graded prime ideal $\mathfrak{p}$ of $R$, the following diagram is commutative up to an isomorphism of functors.

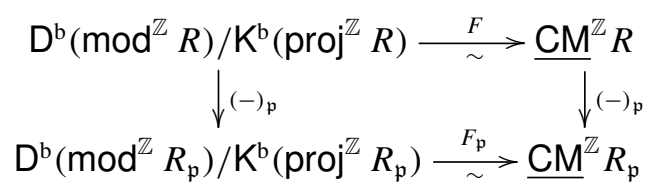


Let $X \in \mathcal{D}_{R}$. For any $\mathfrak{p} \neq R_{>0}$, we have $X_{\mathfrak{p}} \in \mathrm{K}^{\mathrm{b}}\left(\operatorname{proj}^{\mathbb{Z}} R_{\mathfrak{p}}\right)$ and hence $F(X)_{\mathfrak{p}}=$ $F_{\mathfrak{p}}\left(X_{\mathfrak{p}}\right)=0$. Thus $F(X) \in \mathrm{CM}_{0}^{\mathbb{Z}} R$ holds, and hence $F$ restricts a fully faithful

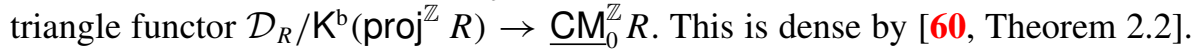

4.2. Basic properties of $\mathbb{Z}$-graded modules. In this subsection, we assume that $R$ is a ring satisfying (R1) and (R2). Recall that $K$ is the $\mathbb{Z}$-graded total quotient ring of $R$. Since $R$ is Cohen-Macaulay, each associated prime ideal of $R$ is minimal. By prime avoidance, there exists a homogeneous non-zero-divisor $r \in R$ with positive degree $p>0$.

We start with the following easy observations.

LEMMA 4.9. (a) The inclusion functor $\operatorname{Mod}^{\geqslant 0} R \rightarrow \operatorname{Mod}^{\mathbb{Z}} R$ has a right adjoint functor $(-)_{\geqslant 0}$.

(b) The restriction functor $\operatorname{Mod}^{\mathbb{Z}} K \rightarrow \operatorname{Mod}^{\mathbb{Z}} R$ has a left adjoint functor $K \otimes_{R}$-.

(c) For any $X \in \bmod ^{\mathbb{Z}} K$, we have $K \otimes_{R}\left(X_{\geqslant 0}\right)=X$.

LEMMA 4.10. We have $K=R\left[r^{-1}\right]$. In particular, for $i \gg 0$, we have $r: R_{i} \simeq$ $R_{i+p}$ and $R_{i}=K_{i}$.

Proof. To prove $K=R\left[r^{-1}\right]$, it suffices to show that each homogeneous non-zerodivisor $x \in K^{\prime}:=R\left[r^{-1}\right]$ is invertible. A bijection between $\mathbb{Z}$-graded prime ideals $\mathfrak{p}$ of $R$ such that $r \notin \mathfrak{p}$ and $\mathbb{Z}$-graded prime ideals of $K^{\prime}$ is given by $\mathfrak{p} \mapsto \mathfrak{p} K^{\prime}$. If $x$ is not invertible, then there exists a $\mathbb{Z}$-graded prime ideal $\mathfrak{p}$ of $R$ such that $x \in \mathfrak{p} K^{\prime}$ and $r \notin \mathfrak{p}$. Since $\mathfrak{p} \subsetneq R_{>0}$ and $\operatorname{dim} R=1, \mathfrak{p}$ is a minimal prime ideal of $R$ and hence consists of zero-divisors, a contradiction to $x \in \mathfrak{p} K^{\prime}$. Thus $K=R\left[r^{-1}\right]$ holds.

Since $R / R r$ is artinian, the remaining assertions follow.

To give basic properties, recall that, for $X, Y \in \bmod ^{\mathbb{Z}} R$ and $n \geqslant 0, \operatorname{Ext}_{R}^{n}(X, Y)$ is a $\mathbb{Z}$-graded $R$-module whose degree $i$ part is $\operatorname{Ext}_{R}^{n}(X, Y)_{i}=\operatorname{Ext}_{\bmod _{R}{ }^{\mathbb{Z}}}^{n}(X, Y(i))$.

Lemma 4.11. (a) We have $R_{a} \subsetneq K_{a}$ and $R_{\geqslant a+1}=K_{\geqslant a+1}$.

(b) For any $i \in \mathbb{Z}$, we have $K(i) \simeq K(i+p)$ and $K(i)_{\geqslant 0} \simeq K(i+p)_{\geqslant 0}$.

(c) For any $i \in \mathbb{Z}, K(i)_{\geqslant 0} \in \bmod ^{\mathbb{Z}} R$ holds.

Proof. (a) Since $\operatorname{Ext}_{\bmod ^{\mathbb{Z}} R}^{1}(k(-a), R)=\operatorname{Ext}_{R}^{1}(k, R)_{a} \neq 0$, there is a nonsplit short exact sequence $0 \rightarrow R \rightarrow X \rightarrow k(-a) \rightarrow 0$. Since $X \in \mathrm{CM}^{\mathbb{Z}} R$, we can regard $X \subset K$ and hence $R_{a} \subsetneq X_{a} \subseteq K_{a}$. 
If $R_{\geqslant a+1} \neq K_{\geqslant a+1}$, then $K / R$ has $k(-i)$ as a simple submodule for some $i \geqslant$ $a+1$. Thus there is a nonsplit short exact sequence $0 \rightarrow R \rightarrow X \rightarrow k(-i) \rightarrow 0$, and hence $\operatorname{Ext}_{R}^{1}(k, R)_{i}=\operatorname{Ext}_{\bmod ^{\mathbb{Z}} R}^{1}(k(-i), R)_{0} \neq 0$, a contradiction.

(b) The multiplication map $r: K(i) \rightarrow K(i+p)$ is an isomorphism.

(c) The assertion follows from (a) and (b).

Now we show the following easy observations.

Proposition 4.12. (a) $K$ is an injective object in $\bmod ^{\mathbb{Z}} K$.

(b) $K(i)_{\geqslant 0}$ is an injective object in $\bmod ^{\geqslant 0} R$ for any $i \in \mathbb{Z}$.

Proof. (a) Let $X \in \bmod ^{\mathbb{Z}} K$. Then we have $X_{\geqslant 0} \in \bmod ^{\mathbb{Z}} R$ by Lemma 4.11(c). Since $\operatorname{dim} R=1$, we have $X_{\geqslant 0} \in \mathrm{CM}^{\mathbb{Z}} R$. Thus $\operatorname{Ext}_{K}^{1}(X, K) \simeq K \otimes_{R} \operatorname{Ext}_{R}^{1}\left(X_{\geqslant 0}\right.$, $R)=K \otimes_{R} 0=0$ by Lemma 4.9(c).

(b) We have isomorphisms of functors on $\bmod ^{\geqslant 0} R$ :

$\operatorname{Hom}_{R}^{\mathbb{Z}}\left(-, K(i)_{\geqslant 0}\right) \stackrel{\text { Lem.4.9(a) }}{=} \operatorname{Hom}_{R}^{\mathbb{Z}}(-, K(i)) \stackrel{\text { Lem.4.9(b) }}{=} \operatorname{Hom}_{K}^{\mathbb{Z}}\left(K \otimes_{R}-, K(i)\right)$.

This is an exact functor since $K$ is a flat $R$-module and $K(i)$ is an injective object in $\bmod ^{\mathbb{Z}} K$ by (a). Thus $K(i)_{\geqslant 0}$ is injective in $\bmod ^{\geqslant 0} R$.

Using an isomorphism $\operatorname{Ext}_{R}^{1}(-, R(a)) \simeq D$ of functors on $\bmod _{0}^{\mathbb{Z}} R \rightarrow \bmod _{0}^{\mathbb{Z}} R$, we show the following key observations.

LEMMA 4.13. (a) For all integers $i, j \in \mathbb{Z}$ satisfying $j<i$ and $j \leqslant a$, we have $\operatorname{Hom}_{R}^{\mathbb{Z}}\left(R(i)_{\geqslant 0}, R(j)_{\geqslant 0}\right)=\operatorname{Hom}_{R}^{\mathbb{Z}}\left(R(i)_{\geqslant 0}, R(j)\right)=0$.

(b) Assume $a \geqslant 0$. For all $i>0$ and $X \in \mathrm{CM}^{\geqslant 0} R$, we have $\underline{\operatorname{Hom}}_{R}^{\mathbb{Z}}\left(R(i)_{\geqslant 0}, X\right)$ $=\operatorname{Hom}_{R}^{\mathbb{Z}}\left(R(i)_{\geqslant 0}, X\right)$.

Proof. (a) The first equality follows from Lemma 4.9(a).

We show the second equality. Consider an exact sequence $0 \rightarrow R(i)_{\geqslant 0} \rightarrow$ $R(i) \rightarrow M \rightarrow 0$ with $M:=R(i) / R(i)_{\geqslant 0} \in \bmod ^{<0} R$. Applying $\operatorname{Hom}_{R}(-, R(j))$, we have an exact sequence

$$
\operatorname{Hom}_{R}^{\mathbb{Z}}(R(i), R(j)) \rightarrow \operatorname{Hom}_{R}^{\mathbb{Z}}\left(R(i)_{\geqslant 0}, R(j)\right) \rightarrow \operatorname{Ext}_{R}^{1}(M, R(j))_{0} .
$$

Since $j<i$, we have $\operatorname{Hom}_{R}^{\mathbb{Z}}(R(i), R(j))=R_{j-i}=0$. Moreover,

$$
\operatorname{Ext}_{R}^{1}(M, R(j))_{0}=\operatorname{Ext}_{R}^{1}(M, R(a))_{j-a}=(D M)_{j-a}=0
$$

holds by $D M \in \bmod ^{>0} R$ and $j-a \leqslant 0$. Thus $\operatorname{Hom}_{R}^{\mathbb{Z}}\left(R(i)_{\geqslant 0}, R(j)\right)=0$ holds. 
(b) Clearly $\operatorname{Hom}_{R}^{\mathbb{Z}}(R(j), X)=0$ for any $j>0$, and $\operatorname{Hom}_{R}^{\mathbb{Z}}\left(R(i)_{\geqslant 0}, R(j)\right)=0$ holds for any $j \leqslant 0$ by (a). Thus the assertion follows.

For $X \in \bmod R$, let $\operatorname{NP}(X):=\left\{\mathfrak{p} \in \operatorname{Spec} R \mid X_{\mathfrak{p}} \notin \operatorname{proj} R_{\mathfrak{p}}\right\}$ be the nonprojective locus of $X$. Clearly, $\operatorname{NP}(X)=\operatorname{Supp}_{\operatorname{Ext}_{R}^{1}}(X, \Omega X)$ holds.

LEMMA 4.14. For $X \in \bmod ^{\mathbb{Z}} R$, each minimal element in $\mathrm{NP}(X)$ is $\mathbb{Z}$-graded. In particular, $X \in$ proj $R$ if and only if $X_{\mathfrak{p}} \in$ proj $R_{\mathfrak{p}}$ for each $\mathbb{Z}$-graded prime ideal $\mathfrak{p}$ of $R$.

Proof. For $\mathfrak{p} \in \operatorname{Spec} R$, we denote by $\mathfrak{p}^{*} \in \operatorname{Spec} R$ the ideal generated by all homogeneous elements in $\mathfrak{p}$. Since $E:=\operatorname{Ext}_{R}^{1}(X, \Omega X)$ is a $\mathbb{Z}$-graded $R$-module, $\mathfrak{p} \in \operatorname{Supp} E$ if and only if $\mathfrak{p}^{*} \in \operatorname{Supp} E[10,1.5 .6]$. Thus each minimal element $\mathfrak{p} \in \operatorname{NP}(X)$ satisfies $\mathfrak{p}=\mathfrak{p}^{*}$.

We give the following description of the category $\mathrm{CM}_{0}^{\mathbb{Z}} R$ in (1.3).

Proposition 4.15. $\mathrm{CM}_{0}^{\mathbb{Z}} R=\left\{X \in \mathrm{CM}^{\mathbb{Z}} R \mid K \otimes_{R} X \in \operatorname{proj} K\right\}$.

Proof. Since $\operatorname{dim} R=1, X \in \mathrm{CM}^{\mathbb{Z}} R$ belongs to $\mathrm{CM}_{0}^{\mathbb{Z}} R$ if and only if $X_{\mathfrak{p}} \in$ proj $R_{\mathfrak{p}}$ for each minimal prime ideal $\mathfrak{p}$ of $R$. Applying Lemma 4.14 to $K \otimes_{R} X \in$ $\bmod ^{\mathbb{Z}} K$, this is equivalent to $K \otimes_{R} X \in$ proj $K$ since $\mathbb{Z}$-graded prime ideals of $K$ correspond bijectively to minimal prime ideals of $R$.

4.3. Proofs of Theorem $\mathbf{1 . 3}$ and Corollaries $\mathbf{1 . 7}$ and $\mathbf{1 . 8}$. Theorem 1.3 follows easily from the following standard observations.

PROPOSITION 4.16. (a) $P=\bigoplus_{i=1}^{p} K(i)$ is a progenerator of $\bmod ^{\mathbb{Z}} K$ such that $\operatorname{End}_{R}^{\mathbb{Z}}(P) \simeq \Lambda$.

(b) There is an equivalence $\operatorname{Hom}_{R}^{\mathbb{Z}}(P,-): \bmod ^{\mathbb{Z}} K \simeq \bmod \Lambda$.

(c) $U=\bigoplus_{i=1}^{p} K(i)_{\geqslant 0}$ is a progenerator in qgr $R$. Therefore $U$ is a tilting object in $\operatorname{per}(\mathrm{qgr} R)$.

(d) $\Lambda$ is a finite-dimensional self-injective k-algebra.

(e) If $R$ is reduced, then $\Lambda$ is a semisimple $k$-algebra. Otherwise, $\Lambda$ has infinite global dimension. 
Proof. (a) Since $\{K(i) \mid i \in \mathbb{Z}\}$ is a progenerator of $\bmod ^{\mathbb{Z}} K$ and $K(i+p) \simeq K(i)$ holds for any $i \in \mathbb{Z}, P$ is a progenerator. Since $\operatorname{End}_{R}(P)=\operatorname{End}_{K}(P)$, we have $\operatorname{End}_{R}^{\mathbb{Z}}(P)=\operatorname{End}_{K}^{\mathbb{Z}}(P) \simeq \Lambda$.

(b) This is immediate from (a) and Morita theory.

(c) Consider the functors $(-)_{\geqslant 0}: \bmod ^{\mathbb{Z}} K \rightarrow \bmod ^{\mathbb{Z}} R$ and $K \otimes_{R}-$ : $\bmod ^{\mathbb{Z}} R \rightarrow \bmod ^{\mathbb{Z}} K$. One can check that they induce mutually quasi-inverse equivalences $\bmod ^{\mathbb{Z}} K \simeq \operatorname{qgr} R$ (for example, [32, Proposition 6.21]). Since $P \in \bmod ^{\mathbb{Z}} K$ corresponds to $U \in \operatorname{qgr} R, U$ is a progenerator in qgr $R$ by (a).

(d) Since $P$ is injective in $\bmod ^{\mathbb{Z}} K$ by Proposition 4.12(a), $\Lambda$ is injective in $\bmod \Lambda$ by (b).

(e) $R$ is reduced if and only if $K$ is reduced if and only if any homogeneous element of $K$ is invertible. This is equivalent to that any object in $\bmod ^{\mathbb{Z}} K$ is projective, that is, gl.dim $\left(\bmod ^{\mathbb{Z}} K\right)=0$. By (b), this is equivalent to that $\Lambda$ is semisimple.

On the other hand, by a classical result of Eilenberg and Nakayama, a selfinjective algebra is either semisimple or of infinite global dimension. Thus the last assertion follows from (d).

We give another proof of Theorem 1.3(a) by using Theorem 3.1. Note that $U$ can be written as

$$
U=\bigoplus_{i=a+1}^{a+p} K(i)_{\geqslant 0}=\bigoplus_{i=a+1}^{a+p} R(i)_{\geqslant 0} \in \mathrm{D}^{\mathrm{b}}\left(\bmod ^{\mathbb{Z}} R\right) .
$$

Theorem 1.3(a) is a direct consequence of the following result.

Proposition 4.17. (a) U belongs to $\mathcal{U}:=\mathcal{D}_{R}^{\geqslant 0} \cap\left(\mathcal{D}_{R}^{>a}\right)^{*}$.

(b) $U$ is a tilting object in $\mathcal{U} \simeq \operatorname{per}($ ggr $R)$.

Proof. (a) Since $U \in \mathcal{D}_{R}^{\geqslant 0}$ holds clearly, we only have to show $U^{*} \in \mathcal{D}_{R}^{>a}$. Fix $i \geqslant a+1$. Since $R(i)_{\geqslant 0} \in \mathrm{CM}^{\mathbb{Z}} R$, we have $\left(R(i)_{\geqslant 0}\right)^{*}=\operatorname{Hom}_{R}\left(R(i)_{\geqslant 0}, R\right)$. Since $\operatorname{Hom}_{R}^{\mathbb{Z}}\left(R(i)_{\geqslant 0}, R(j)\right)=0$ holds for any $j \leqslant a$ by Lemma 4.13(a), we have $\left(R(i)_{\geqslant 0}\right)^{*} \in \mathcal{D}_{R}^{>a}$ as desired.

(b) $\mathcal{U} \simeq \operatorname{per}($ ggr $R)$ holds by Theorem 3.1(b). We have $\operatorname{Hom}_{\mathcal{U}}\left(K(i)_{\geqslant 0}\right.$, $\left.K(j)_{\geqslant 0}[\ell]\right)=0$ for all $\ell \neq 0$ by Proposition 4.12(b). It remains to show $\mathcal{U}=$ thick $U$ or, equivalently, $\operatorname{per}(\operatorname{qgr} R)=\operatorname{thick} U$. For all $i \in \mathbb{Z}$, the multiplication map $r: R(i) \rightarrow R(i+p)$ is an isomorphism in qgr $R$ since $r$ is a non-zero-divisor and hence $R / r R$ is artinian. For all $i$ with $a<i \leqslant a+p, R(i)$ belongs to thick $U$ since $R(i) \simeq R(i)_{\geqslant 0}$ holds in $\operatorname{qgr} R$. Thus $\left.\operatorname{per}(\operatorname{qgr} R)=\operatorname{thick}_{(\operatorname{proj}}{ }^{\mathbb{Z}} R\right)=$ thick $U$ holds. 
Now we prove Corollaries 1.7 and 1.8.

Proof of Corollary 1.7. (a) The isomorphism classes of indecomposable projective objects in qgr $R$ are given by $K^{i}(j)$ with $1 \leqslant i \leqslant m$ and $0 \leqslant j<p_{i}$. Thus their number is $\sum_{i=1}^{m} p_{i}$.

(b) This follows immediately from (a) and Corollary 3.2 since the Grothendieck groups of $\mathrm{K}^{\mathrm{b}}\left(\right.$ proj $\left.^{[\ell, \ell-a-1]} A\right)$ for $a<0$ and $\mathrm{D}^{\mathrm{b}}\left(\bmod ^{[\ell-a, \ell-1]} A\right)$ for $a>0$ are $\mathbb{Z}^{|a|}$.

Proof of Corollary 1.8. Let $k=A_{0}$ and $k[t]$ be a polynomial ring with $\operatorname{deg} t=a$. Then $R=k[t] \otimes_{k} A$ is a $\mathbb{Z}$-graded ring satisfying (R1) and (R2), and the $a$ invariant of $R$ is 0 by [10, Corollary 3.6.14]. By Corollary 3.2 and Theorem 1.3, we have a triangle equivalence $\mathrm{CM}_{0}^{\mathbb{Z}} R \simeq \operatorname{per}($ qgr $R) \simeq \mathrm{K}^{\mathrm{b}}(\operatorname{proj} \Lambda)$ for $\Lambda$ in (1.4) with $p=a$. Since $K=k\left[t^{ \pm 1}\right] \otimes_{k} A$, it is clear that there is an equivalence $\operatorname{proj}^{\mathbb{Z} / a \mathbb{Z}} A \simeq \operatorname{proj} \Lambda$ sending $A(i)$ to the projective $\Lambda$-module given by its $i$ th row (see [37, Theorem 3.1]). Thus $\underline{C M}_{0}^{\mathbb{Z}} R \simeq \mathrm{K}^{\mathrm{b}}(\operatorname{proj} \Lambda) \simeq \mathrm{K}^{\mathrm{b}}\left(\operatorname{proj}^{\mathbb{Z} / a \mathbb{Z}} A\right)$.

4.4. Proofs of Theorem 1.4 and Corollary 1.5. In this subsection, we assume that the $a$-invariant $a$ of $R$ is nonnegative unless otherwise stated. Let

$$
\mathcal{V}:=\mathcal{D}_{R}^{\geqslant-a} \cap\left(\mathcal{D}_{R}^{>a}\right)^{*} \supseteq \mathcal{U}=\mathcal{D}_{R}^{\geqslant 0} \cap\left(\mathcal{D}_{R}^{>a}\right)^{*}
$$

Then we have

$$
\underline{\mathrm{CM}}_{0}^{\mathbb{Z}} R \simeq \mathcal{D}_{R} / \mathrm{K}^{\mathrm{b}}\left(\operatorname{proj}^{\mathbb{Z}} R\right) \stackrel{\text { Thm.3.1(a) }}{\simeq} \mathcal{V} \stackrel{\text { Thm.3.1(c) }}{=} \mathcal{U} \perp \mathrm{D}^{\mathrm{b}}\left(\bmod ^{[-a,-1]} R\right) .
$$

We define a subalgebra of the $a \times a$ matrix algebra $\mathrm{M}_{a}(R)$ by

$$
R^{a}:=\left(R_{i-j}\right)_{1 \leqslant i, j \leqslant a} .
$$

PROPOSITION 4.18. The category $\bmod ^{[-a,-1]} R$ is equivalent to $\bmod R^{a}$ and has a progenerator $\bigoplus_{i=1}^{a}\left(R / R_{\geqslant i}\right)(i) \in \bmod ^{[-a,-1]} R$. Thus $\mathrm{D}^{\mathrm{b}}\left(\bmod ^{[-a,-1]} R\right)$ has a tilting object

$$
W:=\bigoplus_{i=1}^{a}\left(R / R_{\geqslant i}\right)(i)[-1] \in \mathrm{D}^{\mathrm{b}}\left(\bmod ^{[-a,-1]} R\right)
$$

Proof. We have an equivalence $\bmod ^{[-a,-1]} R \simeq \bmod R^{a}$ sending $\bigoplus_{i=-a}^{-1} X_{i}$ to $\left[\begin{array}{llll}X_{-1} & X_{-2} & \cdots & X_{-a}\end{array}\right]$. Since it sends $\bigoplus_{i=1}^{a}\left(R / R_{\geqslant i}\right)(i)$ to $R^{a}$, the first assertion follows. The second assertion is an immediate consequence.

We can glue the tilting objects $U \in \mathcal{U}$ and $W \in \mathrm{D}^{\mathrm{b}}\left(\bmod ^{[-a,-1]} R\right)$ as follows.

LEMMA 4.19. $\mathcal{V}=\mathcal{U} \perp \mathrm{D}^{\mathrm{b}}\left(\bmod ^{[-a,-1]} R\right)$ has a tilting object $U \oplus W$. 
Proof. Clearly $\mathcal{U}=$ thick $U$ and $\mathrm{D}^{\mathrm{b}}\left(\bmod ^{[-a,-1]} R\right)=$ thick $W$ imply $\mathcal{V}=$ thick $(U \oplus W)$.

By Propositions 4.17 and 4.18, we have $\operatorname{Hom}_{\mathcal{V}}(W, W[\ell])=0$ and $\operatorname{Hom}_{\mathcal{V}}(U$, $U[\ell])=0$ for all $\ell \neq 0$. Since $\mathcal{V}=\mathcal{U} \perp \mathrm{D}^{\mathrm{b}}\left(\bmod ^{[-a,-1]} R\right)$, we have $\operatorname{Hom}_{\mathcal{V}}(U$, $W[\ell])=0$ for all $\ell \in \mathbb{Z}$.

It remains to check $\operatorname{Hom}_{\mathcal{V}}\left(\left(R / R_{\geqslant j}\right)(j)[-1], K(i)_{\geqslant 0}[\ell]\right)=0$ for all $\ell \neq 0$. If $\ell<-1$, then this is clear since $\left(R / R_{\geqslant j}\right)(j)$ and $K(i)_{\geqslant 0}$ are modules. If $\ell=-1$, then this follows from $\left(R / R_{\geqslant j}\right)(j) \in \bmod _{0}^{\mathbb{Z}} R$ and $K(i)_{\geqslant 0} \in \mathrm{CM}^{\mathbb{Z}} R$. Assume $\ell>0$. Since the syzygy of $\left(R / R_{\geqslant j}\right)(j)$ is $R(j)_{\geqslant 0}$, we have

$$
\operatorname{Hom}_{\mathcal{V}}\left(\left(R / R_{\geqslant j}\right)(j)[-1], K(i)_{\geqslant 0}[\ell]\right)=\operatorname{Ext}_{R}^{\ell}\left(R(j)_{\geqslant 0}, K(i)_{\geqslant 0}\right)_{0} \stackrel{\text { Prop.4.12(b) }}{=} 0 .
$$

We are ready to prove Theorem 1.4.

Proof of Theorem 1.4. (a) This follows from Lemma 4.19 since $V \simeq U \oplus W$ in $\mathrm{CM}_{0}^{\mathbb{Z}} R$.

(b) This is immediate from (a) and Proposition 4.2.

(c) The triangle equivalence $\mathcal{V} \simeq \underline{\mathrm{CM}}_{0}^{\mathbb{Z}} R$ sends $\bigoplus_{i=1}^{a}\left(R / R_{\geqslant i}\right)(i)[-1]$ to $\bigoplus_{i=1}^{a} R(i)_{\geqslant 0}$. Thus

$$
\operatorname{End}_{R}^{\mathbb{Z}}\left(\bigoplus_{i=1}^{a} R(i)_{\geqslant 0}\right) \simeq \operatorname{End}_{R}^{\mathbb{Z}}\left(\bigoplus_{i=1}^{a}\left(R / R_{\geqslant i}\right)(i)\right)=R^{a}
$$

Hence the left upper entries of (1.6) are correct. The right upper entries are also correct since $\operatorname{Hom}_{R}^{\mathbb{Z}}\left(R(i)_{\geqslant 0}, R(j)_{\geqslant 0}\right)=0$ holds for all $j \leqslant a<i$ by Lemma 4.13(a). Finally, the lower entries are correct since for all $a+1 \leqslant j \leqslant$ $a+p$, we have

$$
\begin{aligned}
\underline{\operatorname{Hom}}_{R}^{\mathbb{Z}}\left(R(i)_{\geqslant 0}, R(j)_{\geqslant 0}\right) & \stackrel{\text { Lem.4.13(b) }}{=} \operatorname{Hom}_{R}^{\mathbb{Z}}\left(R(i)_{\geqslant 0}, R(j)_{\geqslant 0}\right) \\
& \stackrel{\text { Lem.4.11(a) }}{=} \operatorname{Hom}_{R}^{\mathbb{Z}}\left(R(i)_{\geqslant 0}, K(j)_{\geqslant 0}\right) \\
& \stackrel{\text { Lem.4.9(a) }}{=} \operatorname{Hom}_{R}^{\mathbb{Z}}\left(R(i)_{\geqslant 0}, K(j)\right) \\
& \stackrel{\text { Lem.4.9(b) }}{=} \operatorname{Hom}_{K}^{\mathbb{Z}}(K(i), K(j))=K_{j-i} .
\end{aligned}
$$

(d) This follows from Theorem 4.7(a).

(e) For the triangular matrix ring $A=\left[\begin{array}{ll}B & 0 \\ M & C\end{array}\right]$, it is well known that max $\{$ gl.dim $B$, gl.dim $C\} \leqslant$ gl.dim $A \leqslant$ gl.dim $B+\operatorname{gl} \cdot \operatorname{dim} C+1$

holds. Applying it repeatedly, we obtain gl.dim $R^{a}<\infty$. Since $\Gamma$ has a form $\left[\begin{array}{ll}R^{a} & 0 \\ M & \Lambda\end{array}\right]$, it follows that gl.dim $\Gamma<\infty$ if and only if gl.dim $\Lambda<\infty$. Thus the assertion follows from Theorem 1.3(e). 
To prove Corollary 1.5 , we prepare the following.

PROpOSITION 4.20. Under the setting (R1) and (R2), if $a<0$ and $R$ is reduced, then $R \simeq k[t]$.

Proof. Since $R$ is reduced, $K$ is a product $k^{1}\left[t_{1}^{ \pm 1}\right] \times \cdots \times k^{m}\left[t_{m}^{ \pm 1}\right]$ of Laurent polynomial algebras over field extensions $k^{i}$ of $k$ [10, Lemma 1.5.7]. Since $a<0$, we have $R=K_{\geqslant 0}$ by Lemma 4.11(a). Thus $K_{0}=R_{0}=k$ holds, and therefore $K=k\left[t^{ \pm 1}\right]$ and $R=K_{\geqslant 0}=k[t]$.

We are ready to prove Corollary 1.5.

Proof of Corollary 1.5. (a) is shown in Proposition 4.20, and (b) and (c) are immediate from Theorem 1.4. Now (d) is clear from the shape of $\Gamma$ in (1.6).

4.5. Proof of Theorem 1.6. We start with the following general result for 'silting reduction' of triangulated categories.

Proposition 4.21 [40, Theorem 4.8]. Let $\mathcal{U}$ be a triangulated category with a silting object $U$. For any $P \in \operatorname{add} U$, the Verdier quotient $\mathcal{U} /$ thick $P$ has a silting object $U$.

In the rest, we assume $a<0$. Now we prove Theorem 1.6.

Proof of Theorem 1.6. (a)(b) Let $\mathcal{U}=\mathcal{D}_{R}^{\geqslant 0} \cap\left(\mathcal{D}_{R}^{>a}\right)^{*}$. By Theorem 3.1(a)(c), there are triangle equivalences

$$
\underline{\mathrm{CM}}_{0}^{\mathbb{Z}} R \simeq \mathcal{D}_{R}^{\geqslant-a} \cap\left(\mathcal{D}_{R}^{>a}\right)^{*} \simeq \frac{\mathcal{U}}{\mathrm{K}^{\mathrm{b}}\left(\operatorname{proj}^{[0,-a-1]} R\right)}=\frac{\mathcal{U}}{\text { thick } P}
$$

for $P=\bigoplus_{i=a+1}^{0} R(i)$. By Proposition 4.16(c), the triangulated category $\mathcal{U}$ has a tilting object $U=\bigoplus_{i=a+1}^{a+p} R(i)_{\geqslant 0}$. Applying Proposition 4.21 to $\mathcal{U}$ and the direct

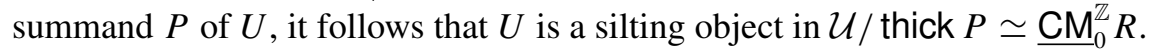

(c) Assume that $R$ is not regular and that $\underline{C M}_{0}^{\mathbb{Z}} R$ has a tilting object $T$. Let $\Lambda=\underline{\operatorname{End}}_{R}^{\mathbb{Z}}(T)$. By Theorem 4.7, there is a triangle equivalence $F: \underline{\mathrm{CM}}_{0}^{\mathbb{Z}} R \simeq$ $\mathrm{K}^{\mathrm{b}}(\operatorname{proj} \Lambda$ ) sending $T$ to $\Lambda$ and making the following diagram commutative.

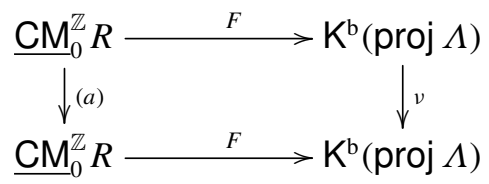


For all $\ell \geqslant 0, v^{\ell}(\Lambda) \in \mathrm{D}^{\leqslant 0}(\bmod \Lambda)$ holds clearly, and hence $H^{i}\left(v^{\ell}(\Lambda)\right)=0$ holds for all $i>0$.

On the other hand, take a surjective morphism $f: \bigoplus_{j=1}^{n} R\left(-b_{j}\right) \rightarrow T$ in $\bmod ^{\mathbb{Z}} R$, and let

$$
s:=\min \left\{b_{j} \mid 1 \leqslant j \leqslant n\right\} \leqslant t:=\max \left\{b_{j} \mid 1 \leqslant j \leqslant n\right\} .
$$

Then $\left(\Omega^{i} T\right)_{<s}=0$ holds for all $i \geqslant 0$. Since $a<0$, there exists $\ell \gg 0$ such that $t<s-\ell a$. Then for all $i \geqslant 0$, we have $\left(\Omega^{i} T(\ell a)\right)_{\leqslant t}=0$ and hence

$\operatorname{Hom}_{R}^{\mathbb{Z}}\left(T, \Omega^{i} T(\ell a)\right) \subset \operatorname{Hom}_{R}^{\mathbb{Z}}\left(\bigoplus_{j=1}^{n} R\left(-b_{j}\right), \Omega^{i} T(\ell a)\right)=\bigoplus_{j=1}^{n}\left(\Omega^{i} T(\ell a)\right)_{b_{j}}=0$.

Thus $H^{-i}\left(v^{\ell}(\Lambda)\right)=\operatorname{Hom}_{\mathrm{D}^{\mathrm{b}}(\bmod \Lambda)}\left(\Lambda, v^{\ell}(\Lambda)[-i]\right)=\underline{\operatorname{Hom}}_{R}^{\mathbb{Z}}\left(T, \Omega^{i} T(\ell a)\right)=0$ holds for all $i \geqslant 0$.

Therefore for $\ell \gg 0, v^{\ell}(\Lambda)$ is acyclic and hence zero in $D^{\mathrm{b}}(\bmod \Lambda)$. This is a contradiction since $v$ is an autoequivalence.

4.6. Proof of Theorem 2.1. (a) Since $\Gamma$ is Iwanaga-Gorenstein by

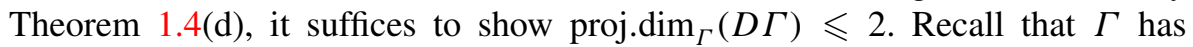
the following form.

$$
\Gamma=\left[\begin{array}{cccccc}
R_{0} & 0 & \cdots & 0 & 0 & 0 \\
R_{1} & R_{0} & \cdots & 0 & 0 & 0 \\
\vdots & \vdots & \ddots & \vdots & \vdots & \vdots \\
R_{a-2} & R_{a-3} & \cdots & R_{0} & 0 & 0 \\
R_{a-1} & R_{a-2} & \cdots & R_{1} & R_{0} & 0 \\
K_{a} & K_{a-1} & \cdots & K_{2} & K_{1} & K_{0}
\end{array}\right] .
$$

For $1 \leqslant i \leqslant a+1$, let $e_{i} \in \Gamma$ be the element whose $(i, i)$-entry is 1 and the others are 0 , and let $P^{i}=\Gamma e_{i}$ be the projective $\Gamma^{\mathrm{op}}$-module corresponding to the $i$ th column.

First, we claim that the simple $\Gamma^{\mathrm{op}}$-module $S^{i}=P^{i} / \operatorname{rad} P^{i}$ has projective dimension at most 2 for $1 \leqslant i \leqslant a$. More precisely, we show that the sequence

$$
0 \rightarrow P^{i+2} \stackrel{{ }^{t}[y-x]}{\longrightarrow}\left(P^{i+1}\right)^{\oplus 2} \stackrel{[x y]}{\longrightarrow} P^{i} \rightarrow S^{i} \rightarrow 0
$$

is exact (where $P^{a+2}=0$ ). Indeed, there is an exact sequence

$$
0 \rightarrow k[x, y](-2) \stackrel{{ }^{t}[y-x]}{\longrightarrow} k[x, y](-1)^{\oplus 2} \stackrel{[x y]}{\longrightarrow} k[x, y] \rightarrow k \rightarrow 0
$$


in $\bmod ^{\mathbb{Z}} k[x, y]$. By $k[x, y]_{<n}=R_{<n}$, the degree $i$-part

$$
0 \rightarrow R_{i+2} \rightarrow\left(R_{i+1}\right)^{\oplus 2} \rightarrow R_{i} \rightarrow 0
$$

of (4.2) is exact for $1 \leqslant i<a=n-2$. Moreover, applying $-\bigotimes_{k[x, y]} K$ to (4.2), we have exact sequences $0 \rightarrow K(-2) \rightarrow K(-1)^{\oplus 2} \rightarrow K \rightarrow 0$ and

$$
0 \rightarrow K_{i+2} \rightarrow\left(K_{i+1}\right)^{\oplus 2} \rightarrow K_{i} \rightarrow 0
$$

for $i \in \mathbb{Z}$. Thus (4.1) is exact since each entry is exact by the above two exact sequences.

By the above claim, any $\Gamma^{\mathrm{op}}$-module annihilated by $e_{a+1}$ has projective dimension at most 2 . In particular, we have proj. $\operatorname{dim}_{\Gamma} D\left(e_{i} \Gamma\right) \leqslant 2$ for $1 \leqslant i \leqslant a$. Finally, there is an exact sequence

$$
0 \rightarrow{ }^{t}\left[\begin{array}{lllll}
0 & 0 & \cdots & 0 & D K_{0}
\end{array}\right] \rightarrow D\left(e_{a+1} \Gamma\right) \rightarrow{ }^{t}\left[D K_{a} D K_{a-1} \cdots D K_{1} 0\right] \rightarrow 0 .
$$

The left term is isomorphic to $\Gamma e_{a+1}$ since $K_{0}$ is a self-injective $k$-algebra. The right term has projective dimension at most 2 since it is annihilated by $e_{a+1}$. Thus proj. $\operatorname{dim}_{\Gamma} D\left(e_{a+1} \Gamma\right) \leqslant 2$ holds, and we have the desired inequality.

(b) Since $R$ is a hypersurface, [2] $=(n)$ holds. Since $R$ has $a$-invariant $n-2$, our triangulated category $\mathrm{CM}_{0}^{\mathbb{Z}} R$ has a Serre functor $\mathbb{S}=(n-2)$ by Proposition 4.6. Thus $\mathbb{S}^{n} \simeq[2(n-2)]$ holds, and $\underline{C M}_{0}^{\mathbb{Z}} R$ is a $\frac{2(n-2)}{n}$-Calabi-Yau triangulated category. If $\Gamma^{\prime}$ is derived equivalent to $\Gamma$, then $\mathrm{K}^{\mathrm{b}}\left(\right.$ proj $\left.\Gamma^{\prime}\right) \simeq{\underline{\mathrm{CM}_{0}^{\mathbb{Z}}}}_{0}$ holds, and hence $1 \leqslant \frac{2(n-2)}{n}<\operatorname{inj} . \operatorname{dim} \Gamma_{\Gamma^{\prime}}^{\prime}$ by [31, Proposition 1.10(c)].

(c) Since $R^{i}=k[x, y] /\left(f_{i}^{n_{i}}\right)$, we have a monomorphism $R \subseteq R^{1} \times \cdots \times R^{m}$ of $\mathbb{Z}$-graded rings whose cokernel has finite length as an $R$-module. Thus we have an isomorphism $K \simeq K^{1} \times \cdots \times K^{m}$ of $\mathbb{Z}$-graded rings. It restricts to an isomorphism $K_{\geqslant 0} \simeq K_{\geqslant 0}^{1} \times \cdots \times K_{\geqslant 0}^{m}$ of $\mathbb{Z}$-graded rings and of $\mathbb{Z}$-graded $R$-modules. We show that $K_{\geqslant 0}^{i}$ is indecomposable in $\mathrm{CM}^{\mathbb{Z}} R$.

By our choice, $g_{i}=\alpha_{i}^{\prime} x+\beta_{i}^{\prime} y \in k[x, y]$ is a non-zero-divisor of $R^{i}$, and by Lemma 4.10, we have $K^{i}=R^{i}\left[g_{i}^{-1}\right]=k\left[h_{i}, g_{i}^{ \pm 1}\right]$ for $h_{i}=f_{i} / g_{i}$. We have isomorphisms

$$
K^{i}=K_{0}^{i}\left[g_{i}^{ \pm 1}\right] \quad \text { and } \quad K_{0}^{i} \simeq k\left[b_{i}\right] /\left(b_{i}^{n_{i}}\right)
$$

for the polynomial ring $k\left[b_{i}\right]$, where $b_{i}$ corresponds to $h_{i}$. Since $\operatorname{End}_{R}^{\mathbb{Z}}\left(K_{\geqslant 0}^{i}\right)=$ $\left(K_{\geqslant 0}^{i}\right)_{0}=K_{0}^{i}=k\left[b_{i}\right] /\left(b_{i}^{n_{i}}\right)$ is a local algebra, $K_{\geqslant 0}^{i}$ is indecomposable. 
(d) The Jacobson radical of $\Gamma$ and its square are

$$
\begin{aligned}
\operatorname{rad} \Gamma= & {\left[\begin{array}{cccccccc}
0 & 0 & 0 & \cdots & 0 & 0 & 0 & 0 \\
R_{1} & 0 & 0 & \cdots & 0 & 0 & 0 & 0 \\
R_{2} & R_{1} & 0 & \cdots & 0 & 0 & 0 & 0 \\
R_{3} & R_{2} & R_{1} & \cdots & 0 & 0 & 0 & 0 \\
\vdots & \vdots & \vdots & \ddots & \vdots & \vdots & \vdots & \vdots \\
R_{a-2} & R_{a-3} & R_{a-4} & \cdots & R_{1} & 0 & 0 & 0 \\
R_{a-1} & R_{a-2} & R_{a-3} & \cdots & R_{2} & R_{1} & 0 & 0 \\
K_{a} & K_{a-1} & K_{a-2} & \cdots & K_{3} & K_{2} & K_{1} & \operatorname{rad} K_{0}
\end{array}\right], } \\
\operatorname{rad}^{2} \Gamma= & {\left[\begin{array}{cccccccc}
0 & 0 & 0 & \cdots & 0 & 0 & 0 & 0 \\
0 & 0 & 0 & \cdots & 0 & 0 & 0 & 0 \\
R_{2} & 0 & 0 & \cdots & 0 & 0 & 0 & 0 \\
R_{3} & R_{2} & 0 & \cdots & 0 & 0 & 0 & 0 \\
\vdots & \vdots & \vdots & \ddots & \vdots & \vdots & \vdots & \vdots \\
R_{a-2} & R_{a-3} & R_{a-4} & \cdots & 0 & 0 & 0 & 0 \\
R_{a-1} & R_{a-2} & R_{a-3} & \cdots & R_{2} & 0 & 0 & 0 \\
K_{a} & K_{a-1} & K_{a-2} & \cdots & K_{3} & K_{2} & \operatorname{rad} K_{1} & \operatorname{rad}^{2} K_{0}
\end{array}\right] . }
\end{aligned}
$$

Thus $\operatorname{rad} \Gamma / \operatorname{rad}^{2} \Gamma$ is a direct sum of the following:

- $e_{i+1}\left(\frac{\operatorname{rad} \Gamma}{\operatorname{rad}^{2} \Gamma}\right) e_{i}=R_{1}=\langle x, y\rangle_{k} \quad(1 \leqslant i \leqslant a-1)$.

- $e_{a+1}\left(\frac{\operatorname{rad} \Gamma}{\operatorname{rad}^{2} \Gamma}\right) e_{a}=\frac{K_{1}}{\operatorname{rad} K_{1}}=\frac{K_{1}^{1}}{\operatorname{rad} K_{1}^{1}} \times \cdots \times \frac{K_{1}^{m}}{\operatorname{rad} K_{1}^{m}}=\frac{k\left[b_{1}\right]}{\left(b_{1}\right)} g_{1} \times \cdots \times \frac{k\left[b_{m}\right]}{\left(b_{m}\right)} g_{m}$.

- $e_{a+1}\left(\frac{\operatorname{rad} \Gamma}{\operatorname{rad}^{2} \Gamma}\right) e_{a+1}=\frac{\operatorname{rad} K_{0}}{\operatorname{rad}^{2} K_{0}}=\frac{\operatorname{rad}_{0}^{1}}{\operatorname{rad}^{2} K_{0}^{1}} \times \cdots \times \frac{\operatorname{rad}_{0}^{m}}{\operatorname{rad}^{2} K_{0}^{m}}=\frac{\left(b_{1}\right)}{\left(b_{1}^{2}\right)} \times \cdots \times \frac{\left(b_{m}\right)}{\left(b_{m}^{2}\right)}$.

Thus we obtain the quiver of $\Gamma$ as in the assertion. The proofs of relations are direct and left to the reader.

(e) This is clear from (d) and (a).

4.7. Proof of Theorem 2.2. We prove Theorem 2.2 by applying Theorem 1.4 and a general recipe to calculate mutation [1] given by Mizuno [58, Theorem 1.2]. We denote by $V$ and $\Gamma$ the tilting object and its endomorphism algebra, respectively, given in Theorem 1.4.

$\left(A_{2 n-1}\right)$ Let $R=k[x, y] /\left(x^{2 n}-y^{2}\right)$ with $\operatorname{deg} x=1$ and $\operatorname{deg} y=n$, so $a=n-1$. Then $K=k\left[t^{ \pm 1}\right] \times k\left[u^{ \pm 1}\right]$ with $\operatorname{deg} t=\operatorname{deg} u=1, x=t+u$ and $y=t^{n}-u^{n}$, so $p=1$. Our $V$ is $\left(\bigoplus_{i=1}^{n-1} R(i)_{\geqslant 0}\right) \oplus k[t] \oplus k[u]$, and $\Gamma$ is the path algebra 
of type $D_{n+1}$ :

$$
R(1) \geqslant 0 \stackrel{x}{\longrightarrow} R(2) \geqslant 0 \stackrel{x}{\longrightarrow} \cdots \frac{x}{\longrightarrow} R(n-2) \geqslant 0 \stackrel{x}{\longrightarrow} R(n-1) \geqslant 0 \stackrel{t}{\longrightarrow}{ }_{k[u] .}^{u}
$$

$\left(A_{2 n}\right)$ Let $R=k[x, y] /\left(x^{2 n+1}-y^{2}\right)$ with $\operatorname{deg} x=2$ and $\operatorname{deg} y=2 n+1$, so $a=2 n-1$. Then $K=k\left[t^{ \pm 1}\right]$ with $\operatorname{deg} t=1, x=t^{2}$ and $y=t^{2 n+1}$, so $p=1$. Our $V$ is $\bigoplus_{i=1}^{2 n} R(i)_{\geqslant 0}$, and $\Gamma$ is the path algebra of type $A_{2 n}$ :

$$
\begin{aligned}
& R(1) \geqslant 0 \stackrel{x}{\longrightarrow} R(3) \geqslant 0 \stackrel{x}{\longrightarrow} \cdots \stackrel{x}{\longrightarrow} R(2 n-3) \geqslant 0 \stackrel{x}{\longrightarrow} R(2 n-1) \geqslant 0 \\
& \downarrow^{t}
\end{aligned}
$$

$\left(D_{2 n+1}\right)$ Let $R=k[x, y] /\left(x^{2 n}-x y^{2}\right)$ with $\operatorname{deg} x=2$ and $\operatorname{deg} y=2 n-1$, so $a=2 n-1$. Then $K=k\left[t^{ \pm 1}\right] \times k\left[u^{ \pm 1}\right]$ with $\operatorname{deg} t=1, \operatorname{deg} u=2 n-1, x=t^{2}$ and $y=t^{2 n-1}+u$, so $p=2 n-1$. Our $V$ is $\left(\bigoplus_{i=1}^{2 n-1} R(i)_{\geqslant 0}\right) \oplus k[t] \oplus\left(\bigoplus_{i=1}^{2 n-1} k[u](i)_{\geqslant 0}\right)$, and $\Gamma$ is

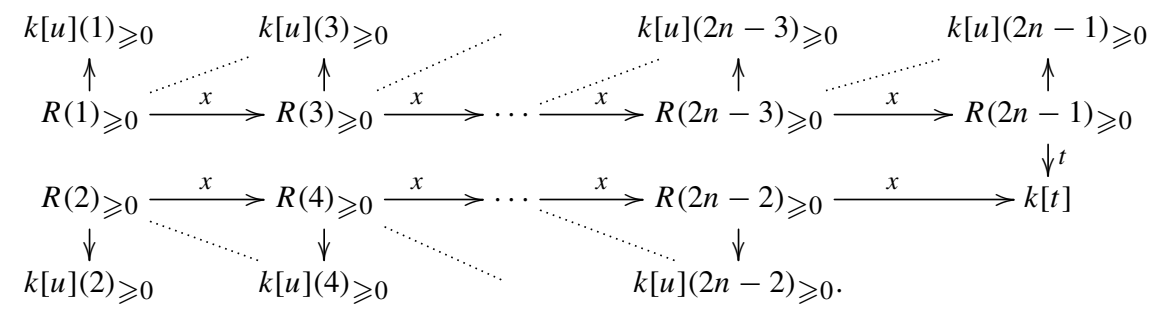

This is derived equivalent to the path algebra of type $A_{4 n-1}$ by mutating the summands $k[u](i)_{\geqslant 0}$ with $1 \leqslant i \leqslant 2 n-1$ :

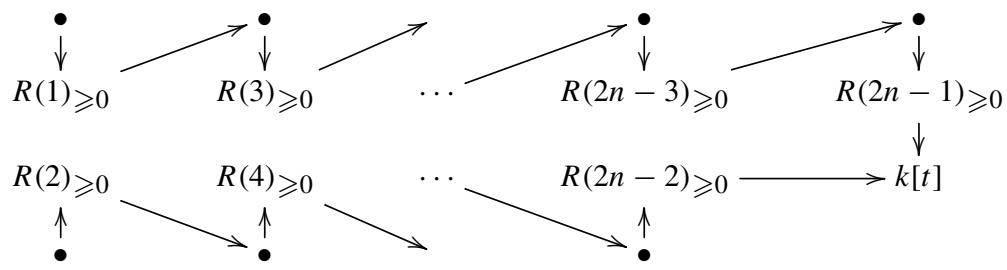

$\left(D_{2 n}\right)$ Let $R=k[x, y] /\left(x^{2 n-1}-x y^{2}\right)$ with $\operatorname{deg} x=1$ and $\operatorname{deg} y=n-1$, so $a=n-1$. Then $K=k\left[t^{ \pm 1}\right] \times k\left[u^{ \pm 1}\right] \times k\left[v^{ \pm 1}\right]$ with $x=t+u, y=t^{n-1}-u^{n-1}+v$, 
$\operatorname{deg} t=\operatorname{deg} u=1$ and $\operatorname{deg} v=n-1$, so $p=n-1$. Our $V$ is $\left(\bigoplus_{i=1}^{n-1} R(i)_{\geqslant 0}\right) \oplus$ $k[t] \oplus k[u] \oplus\left(\bigoplus_{i=1}^{n-1} k[v](i)_{\geqslant 0}\right)$, and $\Gamma$ is

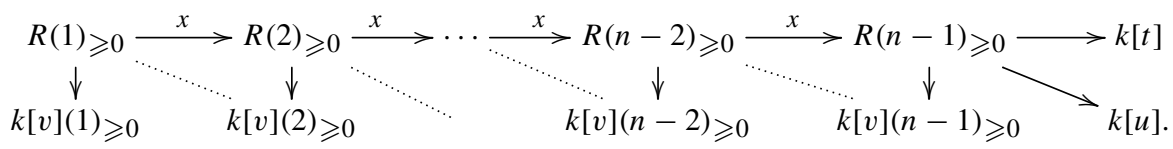

This is derived equivalent to the path algebra of type $D_{2 n}$ by mutating the summands $k[v](i)_{\geqslant 0}$ with $1 \leqslant i \leqslant n-1$ :

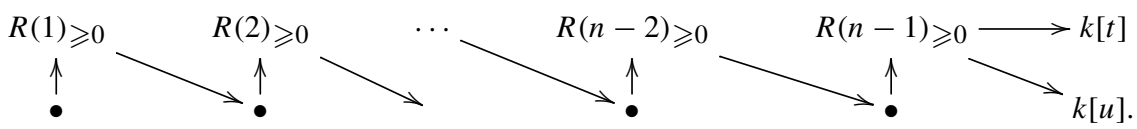

$\left(E_{6}\right)$ Let $R=k[x, y] /\left(x^{4}-y^{3}\right)$ with $\operatorname{deg} x=3$ and $\operatorname{deg} y=4$, so $a=5$. Then $K=k\left[t^{ \pm 1}\right]$ with $\operatorname{deg} t=1, x=t^{3}$ and $y=t^{4}$, so $p=1$. Our $V$ is $\bigoplus_{i=1}^{6} R(i)_{\geqslant 0}$, and $\Gamma$ is

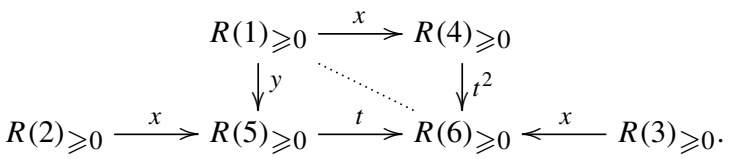

This is derived equivalent to the path algebra of type $E_{6}$ by mutating the summand $R(1)_{\geqslant 0}$ :

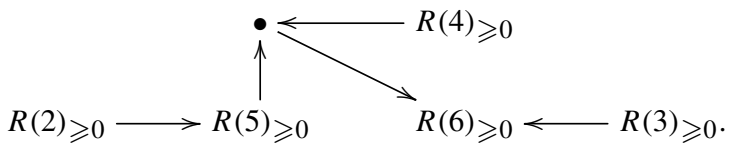

$\left(E_{7}\right)$ Let $R=k[x, y] /\left(x^{3} y-y^{3}\right)$ with $\operatorname{deg} x=2$ and $\operatorname{deg} y=3$, so $a=4$. Then $K=k\left[t^{ \pm 1}\right] \times k\left[u^{ \pm 1}\right]$ with $\operatorname{deg} t=1, \operatorname{deg} u=2, x=t^{2}+u$ and $y=t^{3}$, so $p=2$. Our $V$ is $\left(\bigoplus_{i=1}^{4} R(i)_{\geqslant 0}\right) \oplus k[t] \oplus k[u] \oplus k[u](1)_{\geqslant 0}$, and $\Gamma$ is

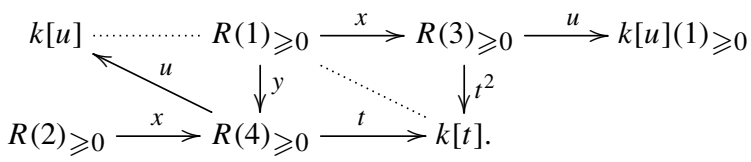

This is derived equivalent to the path algebra of type $E_{7}$ by successively mutating the summands $R(1)_{\geqslant 0}$ and $k[u]$ :

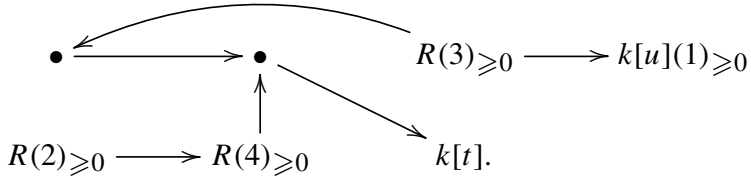


$\left(E_{8}\right)$ Let $R=k[x, y] /\left(x^{5}-y^{3}\right)$ with $\operatorname{deg} x=3$ and $\operatorname{deg} y=5$, so $a=7$. Then $K=k\left[t^{ \pm 1}\right]$ with $\operatorname{deg} t=1, x=t^{3}$ and $y=t^{5}$, so $p=1$. Our $V$ is $\bigoplus_{i=1}^{8} R(i)_{\geqslant 0}$, and $\Gamma$ is

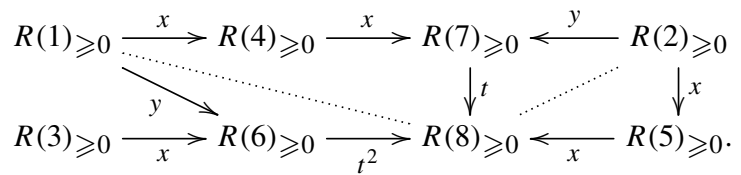

This is derived equivalent to the path algebra of type $E_{8}$ by successively mutating the summands $R(1)_{\geqslant 0}, R(4)_{\geqslant 0}, R(8)_{\geqslant 0}$ and $R(2)_{\geqslant 0}$ :

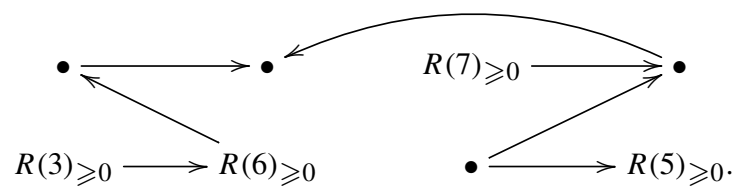

4.8. Proof of Proposition 2.4. We prove Proposition 2.4 by applying Theorem 1.4 and mutation $[\mathbf{1 , 5 8}$. We omit the details of calculations.

(a) We apply Theorem 2.1. Let $K^{i}$ be the $\mathbb{Z}$-graded total quotient ring of $k[x$, $y] /\left(x-\alpha_{i} y\right)$. Since $a=2$ in this case, our $V$ is $\left(\bigoplus_{i=1}^{2} R(i)_{\geqslant 0}\right) \oplus\left(\bigoplus_{i=1}^{4} K_{\geqslant 0}^{i}\right)$, and $\Gamma$ is presented by the quiver with relations

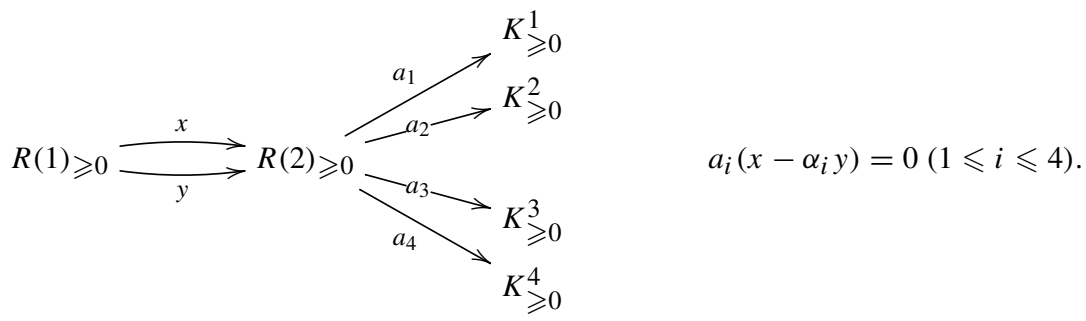

By mutating the summand $R(2)_{\geqslant 0}$, we obtain a tilting object in $\underline{\mathrm{CM}}^{\mathbb{Z}} R$ whose endomorphism algebra is the following canonical algebra, where $\lambda=\left(\alpha_{1}-\right.$ $\left.\alpha_{4}\right)\left(\alpha_{2}-\alpha_{4}\right)\left(\alpha_{1}-\alpha_{3}\right)^{-1}\left(\alpha_{2}-\alpha_{4}\right)^{-1}$ (see also [43, Figure 1.1]).

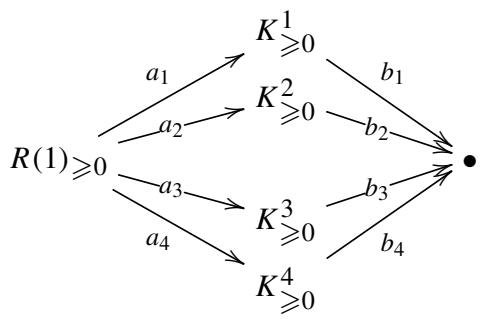

$$
\begin{gathered}
b_{1} a_{1}+b_{2} a_{2}+b_{3} a_{3}=0 \\
b_{1} a_{1}+\lambda b_{2} a_{2}+b_{4} a_{4}=0 .
\end{gathered}
$$


(b) Let $K^{i}$ be the $\mathbb{Z}$-graded total quotient ring of $k[x, y] /\left(x-\alpha_{i} y^{2}\right)$. Since $a=3$ in this case, our $V$ is $\left(\bigoplus_{i=1}^{3} R(i)_{\geqslant 0}\right) \oplus\left(\bigoplus_{i=1}^{3} K_{\geqslant 0}^{i}\right)$, and $\Gamma$ is presented by the quiver with relations

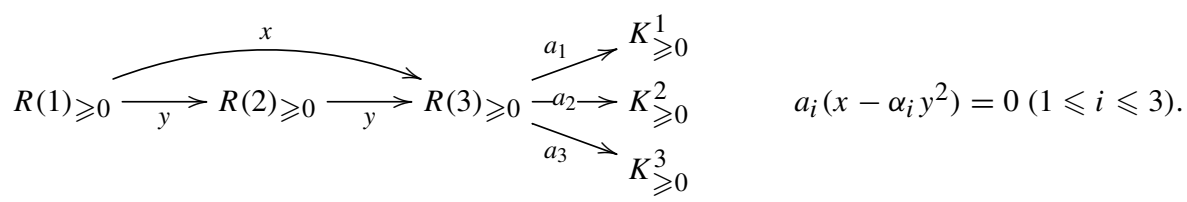

By mutating the summand $R(2)_{\geqslant 0}$, we obtain a tilting object in $\underline{\mathrm{CM}}^{\mathbb{Z}} R$ whose endomorphism algebra is presented by the quiver with relations

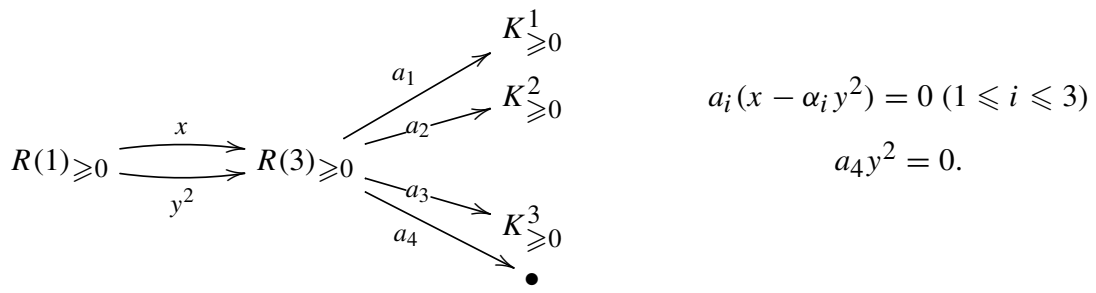

As in case (a), by mutating the summand $R(3)_{\geqslant 0}$, we obtain a tilting object in $\mathrm{CM}^{\mathbb{Z}} R$ whose endomorphism algebra is the following canonical algebra, where $\bar{\lambda}=\left(\alpha_{2}-\alpha_{3}\right)\left(\alpha_{1}-\alpha_{3}\right)^{-1}$.

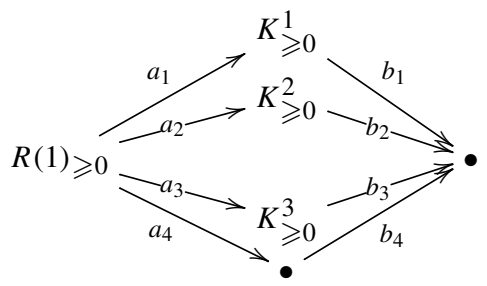

$$
\begin{aligned}
b_{1} a_{1}+b_{2} a_{2}+b_{3} a_{3} & =0 \\
b_{1} a_{1}+\lambda b_{2} a_{2}+b_{4} a_{4} & =0 .
\end{aligned}
$$

4.9. Proof of Proposition 2.5. We start with the following calculation of DG algebras.

Proposition 4.22. Let $\Lambda$ be the algebra in Proposition 2.5(b), and $P$ the projective $\Lambda$-module in Proposition 2.5(d). Then there is a triangle equivalence $\mathrm{K}^{\mathrm{b}}(\operatorname{proj} \Lambda) /$ thick $P \simeq \operatorname{per} k[w] /\left(w^{2}\right)$ for the DG algebra in Proposition 2.5(e).

Proof. Let $M$ be the complex

$$
\cdots \rightarrow 0 \rightarrow P^{1} \stackrel{z}{\rightarrow} P^{2} \stackrel{z}{\rightarrow} \cdots \stackrel{z}{\rightarrow} P^{n-2} \stackrel{z}{\rightarrow} P^{n-1} \stackrel{z}{\rightarrow} P^{n} \rightarrow 0 \rightarrow \cdots
$$


in $\mathrm{K}^{\mathrm{b}}(\operatorname{proj} \Lambda)$ whose nonzero degrees are $1-n, 2-n, \ldots, 0$. Then $\operatorname{Hom}_{K^{\mathrm{b}}(\operatorname{proj} \Lambda)}(P[i], M)=0$ holds for any $i \in \mathbb{Z}$, and there is a triangle $N \rightarrow P^{n} \rightarrow M \rightarrow N[1]$ with $N \in$ thick $P$. Thus we have

$$
\mathrm{K}^{\mathrm{b}}(\operatorname{proj} \Lambda)=\operatorname{thick}\left(P \oplus P^{n}\right)=\operatorname{thick}(P \oplus M)=(\text { thick } P) \perp(\text { thick } M),
$$

and therefore $\mathrm{K}^{\mathrm{b}}(\operatorname{proj} \Lambda) /$ thick $P \simeq$ thick $M$. By [47, Theorem 4.3], we have a triangle equivalence thick $M \simeq \operatorname{per} \mathcal{E} \operatorname{nd}_{\Lambda}(M)$ for the endomorphism DG algebra $\mathcal{E} \operatorname{nd}_{\Lambda}(M)$ of $M$. One can easily verify

$$
\mathcal{E n d}_{\Lambda}(M)^{i}= \begin{cases}\operatorname{Hom}_{\Lambda}\left(P^{n}, P^{1}\right) & (i=1-n) \\ \bigoplus_{i=1}^{n} \operatorname{End}_{\Lambda}\left(P^{i}\right) & (i=0) \\ \bigoplus_{i=1}^{n-1} \operatorname{Hom}_{\Lambda}\left(P^{i}, P^{i+1}\right) & (i=1) \\ 0 & \text { (otherwise) }\end{cases}
$$

and there is a quasi-isomorphism $k[w] /\left(w^{2}\right) \rightarrow \mathcal{E} \operatorname{nd}_{\Lambda}(M)$ of DG algebras given by $w \mapsto\left(z: P^{n} \rightarrow P^{1}\right)$. Thus

$$
\mathrm{K}^{\mathrm{b}}(\operatorname{proj} \Lambda) / \text { thick } P \simeq \text { thick } M \simeq \operatorname{per} \mathcal{E}_{\Lambda}(M) \simeq \operatorname{per} k[w] /\left(w^{2}\right) .
$$

We are ready to prove Proposition 2.5.

Proof of Proposition 2.5. (a) is clear. (b) is shown in Theorem 1.3. (c) and (f) are well known. (d) and the first sentence of (e) are shown in Theorems $1.4(n=1)$ and $1.6(n \geqslant 2)$. The last sentence of (e) follows from Proposition 4.22.

\section{Acknowledgements}

The authors would like to thank Tokuji Araya, Martin Herschend and Ryo Takahashi for useful discussions at the first stage of this project. They also thank Shiro Goto, Helmut Lenzing, Atsushi Takahashi and Yuji Yoshino for valuable discussions. They thank the Centre de Recerca Matemàtica and the organizers of the Workshop ' $(\mathrm{Re})$ emerging methods in Commutative Algebra and Representation Theory' in February 2015, where a part of this collaboration was done. They thank the referee for valuable suggestions.

The second author was partially supported by JSPS Grant-in-Aid for Scientific Research (B) 24340004, (B) 16H03923, (C) 23540045 and (S) 15H05738. The third author was partially supported by Grant-in-Aid for JSPS Research Fellow 13J01461 and JSPS Grant-in-Aid for Young Scientists (B) 26800007. 


\section{Conflict of Interest: None.}

\section{References}

[1] T. Aihara and O. Iyama, 'Silting mutation in triangulated categories', J. Lond. Math. Soc. 85(3) (2012), 633-668.

[2] C. Amiot, O. Iyama and I. Reiten, 'Stable categories of Cohen-Macaulay modules and cluster categories', Amer. J. Math. 137(3) (2015), 813-857.

[3] L. Angeleri Hügel, D. Happel and H. Krause, Handbook of Tilting Theory, London Mathematical Society Lecture Note Series, 332 (Cambridge University Press, Cambridge, 2007).

[4] T. Araya, 'Exceptional sequences over graded Cohen-Macaulay rings', Math. J. Okayama Univ. 41 (1999), 81-102 (2001).

[5] M. Auslander, 'Functors and morphisms determined by objects', in Representation Theory of Algebras (Proc. Conf., Temple Univ., Philadelphia, PA, 1976), Lecture Notes in Pure and Applied Mathematics, 37 (Dekker, New York, 1978), 1-244.

[6] M. Auslander and I. Reiten, 'Almost split sequences for Z-graded rings', in Singularities, Representation of Algebras, and Vector Bundles (Lambrecht, 1985), Lecture Notes in Mathematics, 1273 (Springer, Berlin, 1987), 232-243.

[7] M. Auslander and I. Reiten, 'Cohen-Macaulay modules for graded Cohen-Macaulay rings and their completions', in Commutative Algebra (Berkeley, CA, 1987), Mathematical Sciences Research Institute Publications, 15 (Springer, New York, 1989), 21-31.

[8] A. A. Beilinson, 'Coherent sheaves on $\mathbf{P}^{n}$ and problems in linear algebra', Funktsional. Anal. i Prilozhen. 12(3) (1978), 68-69.

[9] I. N. Bernstein, I. M. Gelfand and S. I. Gelfand, 'Algebraic vector bundles on $\mathbf{P}^{n}$ and problems of linear algebra', Funktsional. Anal. i Prilozhen. 12(3) (1978), 66-67.

[10] W. Bruns and J. Herzog, Cohen-Macaulay Rings, Cambridge Studies in Advanced Mathematics, 39 (Cambridge University Press, Cambridge, 1993).

[11] R.-O. Buchweitz, 'Maximal Cohen-Macaulay modules and Tate-cohomology over Gorenstein rings'. Preprint.

[12] R.-O. Buchweitz, G.-M. Greuel and F.-O. Schreyer, 'Cohen-Macaulay modules on hypersurface singularities. II', Invent. Math. 88(1) (1987), 165-182.

[13] X. Chen, 'Generalized Serre duality', J. Algebra 328 (2011), 268-286.

[14] C. Curtis and I. Reiner, Methods of Representation Theory. Vol. I. With Applications to Finite Groups and Orders, Pure and Applied Mathematics (A Wiley-Interscience Publication, John Wiley \& Sons, Inc., New York, 1981).

[15] L. Demonet and X. Luo, 'Ice quivers with potential associated with triangulations and CohenMacaulay modules over orders', Trans. Amer. Math. Soc. 368(6) (2016), 4257-4293.

[16] L. Demonet and X. Luo, 'Ice quivers with potential arising from once-punctured polygons and Cohen-Macaulay modules', Publ. Res. Inst. Math. Sci. 52(2) (2016), 141-205.

[17] E. Dieterich and A. Wiedemann, 'The Auslander-Reiten quiver of a simple curve singularity', Trans. Amer. Math. Soc. 294(2) (1986), 455-475.

[18] Y. A. Drozd and G.-M. Greuel, 'Cohen-Macaulay module type', Compos. Math. 89(3) (1993), 315-338.

[19] J. A. Drozd and A. V. Router, 'Commutative rings with a finite number of indecomposable integral representations', Izv. Akad. Nauk SSSR Ser. Mat. 31 (1967), 783-798.

[20] D. Eisenbud, 'Homological algebra on a complete intersection, with an application to group representations', Trans. Amer. Math. Soc. 260 (1980), 35-64. 
[21] M. Futaki and K. Ueda, 'Homological mirror symmetry for Brieskorn-Pham singularities', Selecta Math. (N.S.) 17(2) (2011), 435-452.

[22] W. Geigle and H. Lenzing, 'A class of weighted projective curves arising in representation theory of finite-dimensional algebras', in Singularities, Representation of Algebras, and Vector Bundles (Lambrecht, 1985), Lecture Notes in Mathematics, 1273 (Springer, Berlin, 1987), 265-297.

[23] W. Geigle and H. Lenzing, 'Perpendicular categories with applications to representations and sheaves', J. Algebra 144(2) (1991), 273-343.

[24] V. Gelinas, 'Conic intersections, Maximal Cohen-Macaulay modules and the Four Subspace problem'. Preprint, 2017, arXiv:1702.06608.

[25] V. Gelinas, 'Betti tables for indecomposable matrix factorizations of $X Y(X-Y)(X-\lambda Y)^{\prime}$. Preprint, 2017, arXiv:1712.07043.

[26] S. Goto and K. Watanabe, 'On graded rings. I', J. Math. Soc. Japan 30(2) (1978), 179-213.

[27] G. M. Greuel and H. Knörrer, 'Einfache Kurvensingularitäten und torsionsfreie Moduln', Math. Ann. 270(3) (1985), 417-425.

[28] D. Happel, Triangulated Categories in the Representation Theory of Finite-dimensional Algebras, London Mathematical Society Lecture Note Series, 119 (Cambridge University Press, Cambridge, 1988).

[29] D. Happel, 'On Gorenstein algebras', in Representation Theory of Finite Groups and Finitedimensional Algebras (Bielefeld, 1991), Progress in Mathematics, 95 (Birkhäuser, Basel, 1991), 389-404.

[30] M. Herschend and O. Iyama, '2-hereditary algebras and 3-Calabi-Yau algebras from hypersurface singularities', in preparation.

[31] M. Herschend, O. Iyama, H. Minamoto and S. Oppermann, 'Representation theory of GeigleLenzing complete intersections', Mem. Amer. Math. Soc., to appear. Preprint, 2014, arXiv:1409.0668.

[32] M. Herschend, O. Iyama and S. Oppermann, ' $n$-representation infinite algebras', Adv. Math. 252 (2014), 292-342.

[33] A. Higashitani and K. Ueyama, 'Combinatorial study of stable categories of graded CohenMacaulay modules over skew quadric hypersurfaces'. Preprint, 2019, arXiv:1910.10612.

[34] H. Hijikata and K. Nishida, 'Bass orders in nonsemisimple algebras', J. Math. Kyoto Univ. 34(4) (1994), 797-837.

[35] D. Huybrechts, 'Fourier-Mukai transforms in algebraic geometry', in Oxford Mathematical Monographs (The Clarendon Press, Oxford University Press, Oxford, 2006).

[36] O. Iyama, 'Tilting Cohen-Macaulay representations', in Proceedings of the International Congress of Mathematicians-Rio de Janeiro 2018. Vol. II, Invited Lectures (World Sci. Publ., Hackensack, NJ, 2018), 125-162.

[37] O. Iyama and B. Lerner, 'Tilting bundles on orders on $P^{d}$ ', Israel J. Math. 211(1) (2016), 147-169.

[38] O. Iyama and S. Oppermann, 'Stable categories of higher preprojective algebras', Adv. Math. 244 (2013), 23-68.

[39] O. Iyama and R. Takahashi, 'Tilting and cluster tilting for quotient singularities', Math. Ann. 356(3) (2013), 1065-1105.

[40] O. Iyama and D. Yang, 'Silting reduction and Calabi-Yau reduction of triangulated categories', Trans. Amer. Math. Soc. 370(11) (2018), 7861-7898.

[41] O. Iyama and D. Yang, 'Quotients of triangulated categories and Equivalences of Buchweitz, Orlov and Amiot-Guo-Keller', Amer. J. Math., to appear. Preprint, 2017, arXiv:1702.04475. 
[42] H. Jacobinski, 'Sur les ordres commutatifs avec un nombre fini de réseaux indécomposables', Acta Math. 118 (1967), 1-31.

[43] G. Jasso, ' $\tau^{2}$-stable tilting complexes over weighted projective lines', Adv. Math. 273 (2015), $1-31$.

[44] B. T. Jensen, A. King and X. Su, 'A categorification of Grassmannian cluster algebras', Proc. Lond. Math. Soc. (3) 113(2) (2016), 185-212.

[45] H. Kajiura, K. Saito and A. Takahashi, 'Matrix factorization and representations of quivers. II. Type $A D E$ case', Adv. Math. 211(1) (2007), 327-362.

[46] H. Kajiura, K. Saito and A. Takahashi, 'Triangulated categories of matrix factorizations for regular systems of weights with $\epsilon=-1$ ', Adv. Math. 220(5) (2009), 1602-1654.

[47] B. Keller, 'Deriving DG categories', Ann. Sci. Éc. Norm. Supér. (4) 27(1) (1994), 63-102.

[48] B. Keller and H. Krause, 'Tilting preserves finite global dimension', Comptes Rendus Math., to appear. Preprint, 2019, arXiv:1911.11749.

[49] B. Keller and D. Vossieck, 'Aisles in derived categories', Bull. Soc. Math. Belg. Ser. A 40(2) (1988), 239-253. Deuxieme Contact Franco-Belge en Algebre (Faulx-les-Tombes, 1987).

[50] Y. Kimura, 'Tilting theory of preprojective algebras and c-sortable elements', J. Algebra $\mathbf{5 0 3}$ (2018), 186-221.

[51] Y. Kimura, 'Tilting and cluster tilting for preprojective algebras and Coxeter groups', Int. Math. Res. Not. IMRN 2019(18) (2019), 5597-5634.

[52] D. Kussin, H. Lenzing and H. Meltzer, 'Triangle singularities, ADE-chains, and weighted projective lines', Adv. Math. 237 (2013), 194-251.

[53] H. Lenzing and J. A. de la Pena, 'Extended canonical algebras and Fuchsian singularities', Math. Z. 268(1-2) (2011), 143-167.

[54] G. Leuschke and R. Wiegand, Cohen-Macaulay Representations, Mathematical Surveys and Monographs, 181 (American Mathematical Society, Providence, RI, 2012).

[55] M. Lu and B. Zhu, 'Singularity categories of Gorenstein monomial algebras'. Preprint, 2017, arXiv:1708.00311.

[56] H. Minamoto and K. Yamaura, 'On finitely graded Iwanaga-Gorenstein algebras and the stable categories of their (graded) Cohen-Macaulay modules', Adv. Math., to appear. Preprint, 2018, arXiv:1812.03746.

[57] J. Miyachi, 'Duality for derived categories and cotilting bimodules', J. Algebra 185(2) (1996), 583-603.

[58] Y. Mizuno, 'APR tilting modules and graded quivers with potential', Int. Math. Res. Not. IMRN 2014(3) (2014), 817-841.

[59] I. Mori and K. Ueyama, 'Stable categories of graded maximal Cohen-Macaulay modules over noncommutative quotient singularities', Adv. Math. 297 (2016), 54-92.

[60] L. O'Carroll and D. Popescu, 'On a theorem of Knörrer concerning Cohen-Macaulay modules', J. Pure Appl. Algebra 152(1-3) (2000), 293-302.

[61] D. O. Orlov, 'Triangulated categories of singularities and D-branes in Landau-Ginzburg models', Proc. Steklov Inst. Math. 3(246) (2004), 227-248.

[62] D. Orlov, 'Derived categories of coherent sheaves and triangulated categories of singularities', in Algebra, Arithmetic, and Geometry: in Honor of Yu. I. Manin. Vol. II, Progress in Mathematics, 270 (Birkhauser Boston Inc., Boston, MA, 2009), 503-531.

[63] I. Reiten and M. Van den Bergh, 'Noetherian hereditary abelian categories satisfying Serre duality', J. Amer. Math. Soc. 15(2) (2002), 295-366.

[64] J. Rickard, 'Morita theory for derived categories', J. Lond. Math. Soc. (2) 39(3) (1989), 436-456. 
[65] C. M. Ringel, Tame Algebras and Integral Quadratic Forms, Lecture Notes in Mathematics, 1099 (Springer, Berlin, 1984).

[66] D. Simson, Linear Representations of Partially Ordered Sets and Vector Space Categories, Algebra, Logic and Applications, 4 (Gordon and Breach Science Publishers, Montreux, 1992).

[67] A. Skowronski, 'Selfinjective algebras: finite and tame type', in Trends in Representation Theory of Algebras and Related Topics, Contemporary Mathematics, 406 (American Mathematical Society, Providence, RI, 2006), 169-238.

[68] S. P. Smith and M. Van den Bergh, 'Noncommutative quadric surfaces', J. Noncommut. Geom. 7(3) (2013), 817-856.

[69] K. Ueda, 'Triangulated categories of Gorenstein cyclic quotient singularities', Proc. Amer. Math. Soc. 136(8) (2008), 2745-2747.

[70] K. Ueda, 'On graded stable derived categories of isolated Gorenstein quotient singularities', J. Algebra 352 (2012), 382-391.

[71] K. Yamaura, 'Realizing stable categories as derived categories', Adv. Math. 248 (2013), 784-819.

[72] Y. Yoshino, Cohen-Macaulay Modules Over Cohen-Macaulay Rings, London Mathematical Society Lecture Note Series, 146 (Cambridge University Press, Cambridge, 1990). 